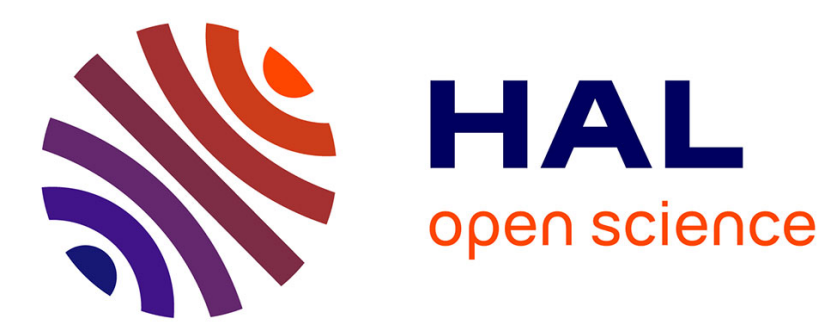

\title{
Relaxation approximation of the Kerr model for the three-dimensional initial-boundary value problem.
}

Gilles Carbou, Bernard Hanouzet

\section{To cite this version:}

Gilles Carbou, Bernard Hanouzet. Relaxation approximation of the Kerr model for the threedimensional initial-boundary value problem.. Journal of Hyperbolic Differential Equations, 2009, 6 (3), pp.577-614. 10.1142/S0219891609001939 . hal-00992606

\section{HAL Id: hal-00992606 https://hal.science/hal-00992606}

Submitted on 29 Mar 2019

HAL is a multi-disciplinary open access archive for the deposit and dissemination of scientific research documents, whether they are published or not. The documents may come from teaching and research institutions in France or abroad, or from public or private research centers.
L'archive ouverte pluridisciplinaire HAL, est destinée au dépôt et à la diffusion de documents scientifiques de niveau recherche, publiés ou non, émanant des établissements d'enseignement et de recherche français ou étrangers, des laboratoires publics ou privés. 


\title{
Relaxation approximation of the Kerr Model for the three dimensional initial-boundary value problem
}

\author{
Gilles Carbou, Bernard Hanouzet \\ Institut de Mathématiques de Bordeaux, UMR 5251 \\ Université Bordeaux 1 \\ 351 cours de la Libération \\ 33405 Talence cedex, France
}

\begin{abstract}
The electromagnetic wave propagation in a nonlinear medium is described by the Kerr model in the case of an instantaneous response of the material, or by the Kerr-Debye model if the material exhibits a finite response time. Both models are quasilinear hyperbolic and are endowed with a dissipative entropy. The initial-boundary value problem with a maximal-dissipative impedance boundary condition is considered here. When the response time is fixed, in both the one-dimensional and two-dimensional transverse electric cases, the global existence of smooth solutions for the Kerr-Debye system is established. When the response time tends to zero, the convergence of the Kerr-Debye model to the Kerr model is established in the general case, i.e. the Kerr model is the zero relaxation limit of the Kerr-Debye model.
\end{abstract}

AMS Classification. 35L50, 35Q60.

Key words. Initial-boundary value problem, Kerr model, Kerr-Debye model, relaxation, nonlinear Maxwell equations.

\section{Introduction.}

Nonlinear Maxwell equations are used for modelling nonlinear optical phenomena and electromagnetic wave propagation is described by

$$
\begin{aligned}
& \partial_{t} D-\operatorname{curl} H=0, \\
& \partial_{t} B+\operatorname{curl} E=0, \\
& \operatorname{div} D=\operatorname{div} B=0 .
\end{aligned}
$$

The field quantities $E$ and $H$ represent the electric and magnetic fields, respectively, while $D$ and $B$ are the electric and magnetic displacements. The constitutive relations are given by

$$
\begin{aligned}
& B=\mu_{0} H, \\
& D=\varepsilon_{0} E+P,
\end{aligned}
$$

where $P$ is the polarization.

We consider here a homogeneous isotropic nonlinear medium (a crystal, for instance), so that the polarization is nonlinear. The Kerr model describes an instantaneous response of the medium and, in this case, $P$ is given by

$$
P=P_{K}=\varepsilon_{0} \varepsilon_{r}|E|^{2} E .
$$

If the medium exhibits a finite response time $\tau$, one should use the Kerr-Debye model for which

$$
P=P_{K D}=\varepsilon_{0} \chi E
$$

where

$$
\partial_{t} \chi+\frac{1}{\tau} \chi=\frac{1}{\tau} \varepsilon_{r}|E|^{2} .
$$

(For further details we refer the reader to, for example, [17] or [20].)

The Kerr-Debye model is a relaxation approximation of the Kerr model where $\tau$ is regarded as the relaxation parameter. (For a general discussion of relaxation problems, see [15].) Formally, when $\tau$ tends to 0, $\chi$ converges to $\varepsilon_{r}|E|^{2}$ and $P_{K D}$ converges to $P_{K}$. 
To cope with a physically realistic situation, we need to cover the initial-boundary value problem (IBVP), although in the rather simple geometry. We assume that the nonlinear material is confined in the region $\left\{x_{1} \geq 0\right\}$, and we set $\left.\Omega=\right] 0,+\infty\left[\times \mathbb{R}^{2}, \Gamma=\{0\} \times \mathbb{R}^{2}\right.$ denoting its boundary. We consider the Kerr and the Kerr-Debye models in the domain $\mathbb{R}_{t}^{+} \times \Omega=\left[0,+\infty\left[\times \Omega\right.\right.$ with the impedance boundary condition on $\mathbb{R}_{t}^{+} \times \Gamma$ and with zero initial data.

Once non-dimensionalized, the initial and boundary value problem (IBVP) for the Kerr model takes the following form (for $(t, x) \in \mathbb{R}^{+} \times \Omega$ ):

$$
\begin{aligned}
& \partial_{t} D-\operatorname{curl} H=0, \\
& \partial_{t} H+\operatorname{curl} E=0,
\end{aligned}
$$

with the constitutive relation

$$
D=\left(1+|E|^{2}\right) E
$$

We suppose that the initial data vanish:

$$
D(0, x)=H(0, x)=0 \quad \text { for } x \in \Omega,
$$

so that the divergence relations hold

$$
\operatorname{div} D=\operatorname{div} H=0 \text { for } t \geq 0 .
$$

We denote by $n={ }^{t}(-1,0,0)$ the outer unit normal along $\Gamma$ and we consider the impedance boundary condition

$$
H \times n+a((E \times n) \times n)=\varphi \text { for }(t, x) \in \mathbb{R}^{+} \times \Gamma,
$$

where $a$ is a positive linear map on $\Gamma$. If $a=I d,(1.2)$ is the classical ingoing wave condition. If $a=0,(1.2)$ is a Dirichlet boundary condition. The system is at rest for $t \leq 0$. It is only excited by the source term $\varphi$ which is localized in the variable $\left(t, x_{2}, x_{3}\right)$ and takes its values in $\Gamma$.

In the same fashion, the IBVP for the Kerr-Debye model (in which $\tau$ is replaced by $\varepsilon$ ) writes (for $(t, x) \in$ $\left.\mathbb{R}^{+} \times \Omega\right)$

$$
\begin{aligned}
& \partial_{t} D_{\varepsilon}-\operatorname{curl} H_{\varepsilon}=0, \\
& \partial_{t} H_{\varepsilon}+\operatorname{curl} E_{\varepsilon}=0, \\
& \partial_{t} \chi_{\varepsilon}=\frac{1}{\varepsilon}\left(\left|E_{\varepsilon}\right|^{2}-\chi_{\varepsilon}\right),
\end{aligned}
$$

with the constitutive relation

$$
D_{\varepsilon}=\left(1+\chi_{\varepsilon}\right) E_{\varepsilon} .
$$

We suppose that the initial data vanish:

$$
D_{\varepsilon}(0, x)=H_{\varepsilon}(0, x)=0, \chi_{\varepsilon}(0, x)=0 \text { for } x \in \Omega,
$$

and we have also

$$
\operatorname{div} D_{\varepsilon}=\operatorname{div} H_{\varepsilon}=0 \text { for } t>0 .
$$

In addition we suppose that we have the same impedance boundary condition

$$
H_{\varepsilon} \times n+a\left(\left(E_{\varepsilon} \times n\right) \times n\right)=\varphi \text { for }(t, x) \in \mathbb{R}^{+} \times \Gamma .
$$

\section{Two-dimensional models.}

Following [19] we can also introduce the two-dimensional transverse magnetic (TM) and transverse electric (TE) models, as follows. For the transverse magnetic case we assume that

$$
\begin{aligned}
& H\left(x_{1}, x_{2}, x_{3}\right)={ }^{t}\left(0, H_{2}\left(x_{1}, x_{3}\right), 0\right), \\
& E\left(x_{1}, x_{2}, x_{3}\right)={ }^{t}\left(E_{1}\left(x_{1}, x_{3}\right), 0, E_{3}\left(x_{1}, x_{3}\right)\right),
\end{aligned}
$$


in the domain $\left(x_{1}, x_{3}\right) \in\left\{x_{1}>0\right\} \times \mathbb{R}$. The Maxwell system becomes

$$
\left\{\begin{array}{l}
\partial_{t} D_{1}+\partial_{3} H_{2}=0 \\
\partial_{t} D_{3}-\partial_{1} H_{2}=0 \\
\partial_{t} H_{2}+\partial_{3} E_{1}-\partial_{1} E_{3}=0
\end{array}\right.
$$

while the divergence-free condition reads

$$
\partial_{1} D_{1}+\partial_{3} D_{3}=0 .
$$

(In this case the divergence condition for $H$ is irrelevant.) The impedance boundary condition reads

$$
H_{2}-a E_{3}=\varphi \text { with } a \geq 0 .
$$

The above system is coupled with either (1.1) (in the Kerr model), or (1.4) and the third equation in (1.3) (in the Kerr-Debye model).

In the transverse electric case, we assume that

$$
\begin{aligned}
& E\left(x_{1}, x_{2}, x_{3}\right)={ }^{t}\left(0, E_{2}\left(x_{1}, x_{3}\right), 0\right), \\
& H\left(x_{1}, x_{2}, x_{3}\right)={ }^{t}\left(H_{1}\left(x_{1}, x_{3}\right), 0, H_{3}\left(x_{1}, x_{3}\right)\right),
\end{aligned}
$$

and we obtain

$$
\left\{\begin{array}{l}
\partial_{t} D_{2}-\partial_{3} H_{1}+\partial_{1} H_{3}=0 \\
\partial_{t} H_{1}-\partial_{3} E_{2}=0 \\
\partial_{t} H_{3}+\partial_{1} E_{2}=0
\end{array}\right.
$$

with the divergence-free condition

$$
\partial_{1} H_{1}+\partial_{3} H_{3}=0 .
$$

The impedance boundary condition becomes

$$
H_{3}+a E_{2}=\varphi \text { with } a \geq 0 .
$$

For the Kerr model, (1.5)-(1.7) is coupled with

$$
D_{2}=\left(1+\left(E_{2}\right)^{2}\right),
$$

and for the Kerr-Debye model, (1.5)-(1.7) is coupled with

$$
\begin{aligned}
& D_{2, \varepsilon}=\left(1+\chi_{\varepsilon}\right) E_{2, \varepsilon}, \\
& \partial_{t} \chi_{\varepsilon}=\frac{1}{\varepsilon}\left(\left(E_{2, \varepsilon}\right)^{2}-\chi_{\varepsilon}\right) .
\end{aligned}
$$

In the case of a fixed finite response time, numerical simulations are presented for these two-dimensional models by finite-difference methods in [19] and by finite-element methods in [13].

\section{One-dimensional model.}

In [3], the following one-dimensional model is introduced:

$$
\begin{aligned}
& E\left(x_{1}, x_{2}, x_{3}\right)={ }^{t}\left(0, e\left(x_{1}\right), 0\right), \\
& H\left(x_{1}, x_{2}, x_{3}\right)={ }^{t}\left(0,0, h\left(x_{1}\right)\right) .
\end{aligned}
$$

In this case, the IBVP for the Kerr model reads

$$
\begin{aligned}
& \partial_{t} d+\partial_{1} h=0, \\
& \partial_{t} h+\partial_{1} e=0, \\
& d=\left(1+e^{2}\right) e,
\end{aligned}
$$


with the impedance boundary condition

$$
h(t, 0)+a e(t, 0)=\varphi(t), t \geq 0, \text { where } a \geq 0,
$$

and the zero initial data

$$
e\left(0, x_{1}\right)=h\left(0, x_{1}\right)=0, x_{1} \geq 0 .
$$

The one-dimensional IBVP for the Kerr-Debye model is

$$
\begin{aligned}
& \partial_{t} d_{\varepsilon}+\partial_{1} h_{\varepsilon}=0, \\
& \partial_{t} h_{\varepsilon}+\partial_{1} e_{\varepsilon}=0, \\
& \partial_{t} \chi_{\varepsilon}=\frac{1}{\varepsilon}\left(\left(e_{\varepsilon}\right)^{2}-\chi_{\varepsilon}\right), \\
& d_{\varepsilon}=\left(1+\chi_{\varepsilon}\right) e_{\varepsilon},
\end{aligned}
$$

with the impedance boundary condition

$$
h_{\varepsilon}(t, 0)+a e_{\varepsilon}(t, 0)=\varphi(t), t \geq 0, \text { where } a \geq 0,
$$

and the null initial data

$$
e_{\varepsilon}\left(0, x_{1}\right)=h_{\varepsilon}\left(0, x_{1}\right)=\chi_{\varepsilon}\left(0, x_{1}\right)=0, x_{1} \geq 0 .
$$

We can also remark that the divergence conditions on $h$ and $d$ are irrelevant for both models.

\section{Mathematical properties and main results.}

Since both Kerr and Kerr-Debye models are endowed with strictly convex entropies, the associated systems are symmetrizable hyperbolic. Furthermore the boundary is characteristic of constant multiplicity (except for the system (1.8)) and the boundary conditions are maximal dissipative. So the general results in [10] ensure the local existence of smooth solutions for smooth data. For the Kerr model a better local existence result is proved in [16]. A similar result for the Kerr-Debye model is established in the Appendix.

We denote by $T^{*}$ and $T_{\varepsilon}^{*}$ the lifespan of such smooth solutions for the Kerr and the Kerr-Debye IBVP, respectively. Since the Kerr model is a homogeneous quasilinear hyperbolic system, shock waves can appear at $T^{*}$. In particular, for the one-dimensional case, we can rewrite (1.8) as a p-system which is genuinely nonlinear for $d \neq 0$. In this case, using the results of [14], we can exhibit initial data such that the lifespan $T^{*}$ is finite with formation of shock waves (see also [4]). On the other hand the Kerr-Debye model is a quasilinear hyperbolic system with source term and it is totally linearly degenerate, i.e. each characteristic field is linearly degenerate. So we can expect that, if the lifespan $T_{\varepsilon}^{*}$ is finite, the behavior of the smooth solution is analogous to the semilinear case. Indeed we obtained this result in the 1-d case in [5]: if $T_{\varepsilon}^{*}$ is finite then the solution and its gradient explode, i.e. shock formation never occurs. In fact, using stronger dissipative properties for the Kerr-Debye model we proved in [7] that $T_{\varepsilon}^{*}=+\infty$ for the one-dimensional Cauchy problem. This global existence result should be also established for the Cauchy problem in the 2-d TE case. In the present paper we choose to prove the same result for the impedance IBVP in the 1-d and 2-d TE cases (see Theorem 1 below). In the proofs we must take into account a new difficulty which does not appear for the Cauchy problem: the boundary $\Gamma$ of the domain $\Omega$ is characteristic.

The Kerr-Debye model is a relaxation approximation of the Kerr model in the sense developed in [8] . The stability conditions in [8] and [18] are satisfied so it is natural to study the behavior of the smooth solutions to the Kerr-Debye model as the relaxation coefficient $\varepsilon$ tends to zero. Concerning the Cauchy problem with initial data $\left(D_{0}, H_{0}, \chi_{0}\right)$ satisfying $\operatorname{div} D_{0}=\operatorname{div} H_{0}=0, \chi_{0} \geq 0$, the convergence for the smooth solutions is proved in [11] using the results of [18]. Generally a boundary layer in time appears because of the non compatibility of the initial data with the equilibrium condition $\chi=|E|^{2}$. We study here the convergence for the impedance IBVP. In this case no boundary layer appears, neither in the time variable (since the zero initial data fit the equilibrium condition) nor in the space variables (since the boundary condition is the same for both IBVP). In the one-dimensional case, we presented in [3] a first convergence result. In fact we obtained the convergence of $(1.11)-(1.12)-(1.13)$ to $(1.8)-(1.9)-(1.10)$ on some interval $[0, \tilde{T}] \subset\left[0, T^{*}[\right.$. The 
same kind of convergence result is announced for the 3 -d case in [6]. Here we improve these results in all cases since the convergence is obtained on each interval $[0, T] \subset\left[0, T^{*}[\right.$.

Our paper is organized as follows. In Section 2 we exhibit general properties of the Kerr and the Kerr-Debye models. For a fixed $\varepsilon$ we establish the global existence result of the solutions to the Kerr Debye 2-d TE model. Section 3 is devoted to convergence results when the relaxation parameter tends to zero.

\section{General Properties.}

\subsection{Properties of the Kerr model.}

We recall the initial-boundary value problem for the general Kerr model:

$$
\left\{\begin{array}{l}
\partial_{t} D-\operatorname{curl} H=0, \\
\partial_{t} H+\operatorname{curl} E=0, \\
D=\left(1+|E|^{2}\right) E,
\end{array}\right.
$$

for $(t, x) \in \mathbb{R}^{+} \times \Omega$ together with the initial and boundary conditions

$$
\begin{gathered}
D(t=0)=H(t=0)=0 \text { for } x \in \Omega, \\
H \times n+a((E \times n) \times n)=\varphi \text { for }(t, x) \in \mathbb{R}^{+} \times \Gamma .
\end{gathered}
$$

The energy density given by

$$
\mathcal{E}_{K}(D, H)=\frac{1}{2}\left(|E|^{2}+|H|^{2}+\frac{3}{2}|E|^{4}\right)
$$

is a strictly convex entropy (with associated flux function $E \times H$ ), so (2.1) is a quasilinear hyperbolic symmetrizable system.

In the three-dimensional case, the eigenvalues are (for $\xi \neq 0)$

$$
\lambda_{1}(E, \xi) \leq \lambda_{2}(E, \xi)<\lambda_{3}=\lambda_{4}=0<\lambda_{5}=-\lambda_{2} \leq \lambda_{6}=-\lambda_{1},
$$

so the boundary $\mathbb{R}^{+} \times \Gamma$ is characteristic of constant multiplicity two. By direct calculations we obtain

$$
\begin{aligned}
& \lambda_{1}(E, \xi)=-\left(1+|E|^{2}\right)^{-\frac{1}{2}}|\xi|, \\
& \lambda_{2}(E, \xi)=-\left(1+|E|^{2}\right)^{-\frac{1}{2}}\left(1+3|E|^{2}\right)^{-\frac{1}{2}}\left(\left(1+|E|^{2}\right)|\xi|^{2}+2(E \cdot \xi)^{2}\right)^{\frac{1}{2}} .
\end{aligned}
$$

In the two-dimensional cases, TM and TE, the eigenvalues are of the form:

$$
\lambda_{1}(E, \xi)<\lambda_{2}=0<\lambda_{3}=-\lambda_{1},
$$

so the boundary $\mathbb{R}^{+} \times \Gamma$ is characteristic of constant multiplicity one. By direct calculations we obtain for the TM model:

$$
\lambda_{1}^{T M}(E, \xi)=-\left(1+|E|^{2}\right)^{-\frac{1}{2}}\left(1+3|E|^{2}\right)^{-\frac{1}{2}}\left(\left(1+|E|^{2}\right)|\xi|^{2}+2(E \cdot \xi)^{2}\right)^{\frac{1}{2}},
$$

and for the TE model:

$$
\lambda_{1}^{T E}(E, \xi)=-\left(1+3\left|E_{2}\right|^{2}\right)^{-\frac{1}{2}}|\xi| .
$$

In the one-dimensional case, the system is strictly hyperbolic and the boundary is non characteristic. We have

$$
\lambda_{1}(E)<0<\lambda_{2}=-\lambda_{1},
$$

with

$$
\lambda_{1}(E)=-\left(1+3 e^{2}\right)^{-\frac{1}{2}} .
$$

We remark that the impedance boundary condition (2.3) is maximal dissipative. Generally speaking local existence results of smooth solutions to IBVP for quasilinear hyperbolic systems with characteristic boundary are available in [10]. For the Kerr system (2.1)-(2.2)-(2.3) we can also apply a more adapted result in [16]. We assume that the source term $\varphi$ is compactly supported in $\mathbb{R}^{+} \times \Gamma$. We denote by $H^{s}$ the classical Sobolev spaces and we suppose that $\varphi$ belongs to $H^{s}\left(\mathbb{R}_{t} \times \Gamma\right)$ for $s$ great enough. So the boundary condition (2.3) and the initial data (2.2) are compatible and by [16] we obtain smooth local solutions. 
Proposition 1 Let $\varphi \in H^{3}(\mathbb{R} \times \Gamma)$ compactly supported in $\mathbb{R}^{+} \times \Gamma$. Then there exists a maximal smooth solution $(D, H)$ to the IBVP (2.1)-(2.2)-(2.3) which lifespan is denoted by $T^{*}$ and such that

$$
\partial_{t}^{i}(D, H) \in \mathcal{C}^{0}\left(\left[0, T^{*}\left[; H^{3-i}(\Omega)\right) \text { for } i=0,1,2,3 .\right.\right.
$$

Remark 1 In the third part we need more regular solutions. In fact, using [10] we obtain the following result: for all $k \in \mathbb{N}$, there exists $s_{0}(k)$ such that if $\varphi \in H^{s_{0}(k)}(\mathbb{R} \times \Gamma)$ then

$$
\partial_{t}^{i}(D, H) \in \mathcal{C}^{0}\left(\left[0, T^{*}\left[; H^{k-i}(\Omega)\right) \text { for } i=0, \ldots, k .\right.\right.
$$

For example, for $k=7$, using theorem 2 in [10] we obtain that $s_{0}(7)=18$.

The previous existence result is stated in the three-dimensional case. Analogous results can be obtained for both the two-dimensional and the one-dimensional cases.

\subsection{Properties of the Kerr-Debye models.}

Consider for a fixed $\varepsilon>0$ the Kerr-Debye model

$$
\left\{\begin{array}{l}
\partial_{t} D_{\varepsilon}-\operatorname{curl} H_{\varepsilon}=0, \\
\partial_{t} H_{\varepsilon}+\operatorname{curl} E_{\varepsilon}=0, \\
\partial_{t} \chi_{\varepsilon}=\frac{1}{\varepsilon}\left(\left|E_{\varepsilon}\right|^{2}-\chi_{\varepsilon}\right), \\
D_{\varepsilon}=\left(1+\chi_{\varepsilon}\right) E_{\varepsilon} .
\end{array}\right.
$$

for $(t, x) \in \mathbb{R}^{+} \times \Omega$ together with the initial and boundary conditions

$$
\begin{gathered}
D_{\varepsilon}(0, x)=H_{\varepsilon}(0, x)=0, \chi_{\varepsilon}(0, x)=0 \text { for } x \in \Omega, \\
H_{\varepsilon} \times n+a\left(\left(E_{\varepsilon} \times n\right) \times n\right)=\varphi \text { for }(t, x) \in \mathbb{R}^{+} \times \Gamma .
\end{gathered}
$$

The divergence free conditions are preserved by the system:

$$
\operatorname{div} H_{\varepsilon}=\operatorname{div}\left(1+\chi_{\varepsilon}\right) E_{\varepsilon}=0 .
$$

By the third equation in (2.4) we observe that we have

$$
\chi_{\varepsilon} \geq 0
$$

The energy density given by

$$
\mathcal{E}_{K D}(D, H, \chi)=\frac{1}{2}(1+\chi)^{-1}|D|^{2}+\frac{1}{2}|H|^{2}+\frac{1}{4} \chi^{2}
$$

is a strictly convex entropy in the domain $\{\chi \geq 0\}$ (with associated flux function $E \times H=(1+\chi)^{-1} D \times H$ ). So (2.4) is a quasilinear symmetrizable hyperbolic system.

In the three-dimensional case the eigenvalues are, for $\xi \neq 0$,

$$
\mu_{1}(\chi, \xi)=\mu_{2}<\mu_{3}=\mu_{4}=\mu_{5}=0<\mu_{6}=\mu_{7}=-\mu_{1},
$$

where $\mu_{1}=-(1+\chi)^{-\frac{1}{2}}|\xi|$. So the boundary is characteristic of constant multiplicity three.

In the two-dimensional cases we obtain

$$
\mu_{1}(\chi, \xi)<\mu_{2}=\mu_{3}=0<\mu_{4}=-\mu_{1}
$$


where $\mu_{1}=-(1+\chi)^{-\frac{1}{2}}|\xi|$. So the boundary is characteristic of constant multiplicity two.

In the one-dimensional case, the system is strictly hyperbolic and the boundary is characteristic of constant multiplicity one. The eigenvalues are

$$
\mu_{1}(\chi)<\mu_{2}=0<\mu_{3}=-\mu_{1},
$$

where $\mu_{1}=-(1+\chi)^{-\frac{1}{2}}$.

We remark that each characteristic field of the quasilinear hyperbolic system (2.4) is linearly degenerate. It suffices to prove this property for the eigenvalue $\mu_{1}(\chi, \xi)=-(1+\chi)^{-\frac{1}{2}}|\xi|$ for which the last component of the corresponding eigenvector vanishes, so we have

$$
\nabla \mu_{1} \cdot r_{1} \equiv 0
$$

In the 3 -d case this property is also obtained by a general result in [2].

Using the special structure of the Kerr-Debye model, we prove in the Appendix the following existence result.

Proposition 2 Let $\varphi \in H^{5}(\mathbb{R} \times \Gamma)$ compactly supported in $\mathbb{R}^{+} \times \Gamma$. Then there exists a maximal smooth solution $\left(D_{\varepsilon}, H_{\varepsilon}, \chi_{\varepsilon}\right)$ to the IBVP (2.4)-(2.5)-(2.6) which lifespan is denoted by $T_{\varepsilon}^{*}$ and such that

$$
\partial_{t}^{i}\left(D_{\varepsilon}, H_{\varepsilon}, \chi_{\varepsilon}\right) \in \mathcal{C}^{0}\left(\left[0, T_{\varepsilon}^{*}\left[; H^{3-i}(\Omega)\right) \text { for } i=0,1,2,3 .\right.\right.
$$

Analogous results can be established in both the 2-d and the 1-d cases.

\subsection{Dissipative properties of the Kerr-Debye models.}

For the Cauchy problem, it is well known (see [11]) that the Kerr-Debye system is dissipative and we have

$$
\frac{d}{d t} \int_{\mathbb{R}^{3}} \mathcal{E}_{K D}(D, H, \chi) d x=-\frac{\varepsilon}{2} \int_{\mathbb{R}^{3}}\left|\partial_{t} \chi\right|^{2} d x
$$

We generalize this dissipative formula for the IBVP (2.4)-(2.5)-(2.6). In addition we exhibit another dissipative property for the time derivatives. To start with we extend the boundary condition (2.6) in the following way: we replace $H_{2}$ by $H_{2}+\varphi_{3}\left(t, x_{2}, x_{3}\right) \eta\left(x_{1}\right), H_{3}$ by $H_{3}-\varphi_{2}\left(t, x_{2}, x_{3}\right) \eta\left(x_{1}\right)$, where $\eta$ is a cut off function which is equal to 1 in a neighbourhood of 0 , compactly supported in the interval [0,1[. Furthermore, to preserve the divergence free condition (2.7) we replace $H_{1}$ by

$$
H_{1}+\left(\partial_{2} \varphi_{3}-\partial_{3} \varphi_{2}\right)\left(t, x_{2}, x_{3}\right) \int_{x_{1}}^{+\infty} \eta(s) d s
$$

and we denote

$$
\mathcal{R}(t, x)=\left(\begin{array}{l}
\left(\partial_{2} \varphi_{3}-\partial_{3} \varphi_{2}\right)\left(t, x_{2}, x_{3}\right) \int_{x_{1}}^{+\infty} \eta(s) d s \\
\varphi_{3}\left(t, x_{2}, x_{3}\right) \eta\left(x_{1}\right) \\
-\varphi_{2}\left(t, x_{2}, x_{3}\right) \eta\left(x_{1}\right)
\end{array}\right) .
$$

Then the system (2.4)-(2.7) becomes in the variable $V=(U, \chi)=(E, H, \chi)$ :

(i) $\quad(1+\chi) \partial_{t} E+\left(\partial_{t} \chi\right) E-\operatorname{curl} H=G_{1}$,

(ii) $\partial_{t} H+\operatorname{curl} E=G_{2}$,

(iii) $\partial_{t} \chi=\frac{1}{\varepsilon}\left(|E|^{2}-\chi\right)$, 
where $G_{1}=\operatorname{curl} \mathcal{R}$ and $G_{2}=-\partial_{t} \mathcal{R}$, with the homogeneous initial and boundary conditions:

$$
\begin{gathered}
V(0, x)=0, x \in \Omega, \\
H \times n+a((E \times n) \times n)=0,(t, x) \in \mathbb{R}^{+} \times \Gamma .
\end{gathered}
$$

The divergence free conditions are preserved:

$$
\operatorname{div} H=\operatorname{div}((1+\chi) E)=0 .
$$

For the IBVP (2.9)-(2.12) the dissipation properties are described by the following result:

Proposition 3 Let $V$ be the smooth solution to (2.9)-(2.12) given by Proposition 2. We denote $E_{T}=$ $\left(0, E_{2}, E_{3}\right)$. Then, on the interval $\left[0, T_{\varepsilon}^{*}[\right.$, we have

$$
\begin{gathered}
\frac{d}{d t} \int_{\Omega} \mathcal{E}_{K D}(D, H, \chi) d x+\frac{\varepsilon}{2} \int_{\Omega}\left|\partial_{t} \chi\right|^{2} d x+\int_{\Gamma} a\left(E_{T}\right) \cdot E_{T} d x_{2} d x_{3}=\int_{\Omega}\left(G_{1} \cdot E+G_{2} \cdot H\right) d x \\
\frac{1}{2} \frac{d}{d t} \int_{\Omega}\left((1+\chi)\left|\partial_{t} E\right|^{2}+\left|\partial_{t} H\right|^{2}+\frac{1}{2}\left(\partial_{t} \chi\right)^{2}\right) d x+\frac{3}{2 \varepsilon} \int_{\Omega}|E|^{2}\left|\partial_{t} E\right|^{2} d x+\frac{\varepsilon}{2} \int_{\Omega}\left|\partial_{t t} \chi\right|^{2} d x \\
\quad+\int_{\Gamma} a\left(\partial_{t} E_{T}\right) \cdot \partial_{t} E_{T} d x_{2} d x_{3}=\frac{3}{2 \varepsilon} \int_{\Omega} \chi\left|\partial_{t} E\right|^{2} d x+\int_{\Omega}\left(\partial_{t} G_{1} \cdot \partial_{t} E+\partial_{t} G_{2} \cdot \partial_{t} H\right) d x
\end{gathered}
$$

Proof. Taking the inner product of (2.9.i) with $E$ and (2.9.ii) with $H$ we obtain

$$
\int_{\Omega}(1+\chi) \partial_{t} E \cdot E d x+\int_{\Omega}|E|^{2} \partial_{t} \chi d x-\int_{\Omega} \operatorname{curl} H \cdot E d x+\int_{\Omega} \partial_{t} H \cdot H d x+\int_{\Omega} \operatorname{curl} E \cdot H d x=\int_{\Omega}\left(G_{1} \cdot E+G_{2} \cdot H\right) d x .
$$

After integrating by parts we obtain

$$
\begin{aligned}
-\int_{\Omega} \operatorname{curl} H \cdot E d x+\int_{\Omega} \operatorname{curl} E \cdot H d x & =\int_{\Gamma}(H \times n) \cdot E d x_{2} d x_{3} \\
& =-\int_{\Gamma} a((E \times n) \times n) \cdot E d x_{2} d x_{3} \\
& =\int_{\Gamma} a\left(E_{T}\right) \cdot E_{T} d x_{2} d x_{3} .
\end{aligned}
$$

In addition, we have

$$
\int_{\Omega}(1+\chi) \partial_{t} E \cdot E d x=\frac{1}{2} \frac{d}{d t} \int_{\Omega}(1+\chi)|E|^{2} d x-\frac{1}{2} \int_{\Omega}|E|^{2} \partial_{t} \chi d x
$$

Using the last equation in (2.9), we obtain

$$
\frac{1}{2} \int_{\Omega}|E|^{2} \partial_{t} \chi d x=\frac{1}{4} \frac{d}{d t} \int_{\Omega}|\chi|^{2} d x+\frac{\varepsilon}{2} \int_{\Omega}\left(\partial_{t} \chi\right)^{2} d x .
$$

So replacing in (2.15) we obtain (2.13).

We differentiate (2.9) and (2.11) with respect to $t$ :

(i) $(1+\chi) \partial_{t t} E+2 \partial_{t} \chi \partial_{t} E+\partial_{t t} \chi E-\operatorname{curl} \partial_{t} H=\partial_{t} G_{1}$,

(ii) $\partial_{t t} H+\operatorname{curl} \partial_{t} E=\partial_{t} G_{2}$,

(iii) $\partial_{t t} \chi=\frac{2}{\varepsilon} E \cdot \partial_{t} E-\frac{1}{\varepsilon} \partial_{t} \chi$, 


$$
\partial_{t} H \times n+a\left(\left(\partial_{t} E \times n\right) \times n\right)=0 \text { in } \mathbb{R}^{+} \times \Gamma .
$$

As before we obtain

$$
\begin{array}{r}
\frac{1}{2} \frac{d}{d t} \int_{\Omega}\left((1+\chi)\left|\partial_{t} E\right|^{2}+\left|\partial_{t} H\right|^{2}\right) d x+\frac{3}{2} \int_{\Omega} \partial_{t} \chi\left|\partial_{t} E\right|^{2} d x+\int_{\Omega} \partial_{t t} \chi E \cdot \partial_{t} E d x+\int_{\Gamma} a\left(\partial_{t} E_{T}\right) \cdot \partial_{t} E_{T} d x_{2} d x_{3} \\
=\int_{\Omega}\left(\partial_{t} G_{1} \cdot \partial_{t} E+\partial_{t} G_{2} \cdot \partial_{t} H\right) d x
\end{array}
$$

The last equation in (2.9) yields

$$
\int_{\Omega} \partial_{t t} \chi E \cdot \partial_{t} E d x=\frac{1}{4} \frac{d}{d t} \int_{\Omega}\left(\partial_{t} \chi\right)^{2} d x+\frac{\varepsilon}{2} \int_{\Omega}\left(\partial_{t t} \chi\right)^{2} d x
$$

so (2.14) holds.

Remark 2 It is clear that (2.14) holds also for the Cauchy problem.

\subsection{Global existence for the 2d TE Kerr-Debye system.}

For the two-dimensional TE model, the system is the following:

$$
\left\{\begin{array}{l}
\partial_{t} D_{2}-\partial_{3} H_{1}+\partial_{1} H_{3}=0 \\
\partial_{t} H_{1}-\partial_{3} E_{2}=0 \\
\partial_{t} H_{3}+\partial_{1} E_{2}=0 \\
\partial_{t} \chi=\frac{1}{\varepsilon}\left(\left|E_{2}\right|^{2}-\chi\right)
\end{array}\right.
$$

for $t>0$, for $\left(x_{1}, x_{3}\right) \in \mathbb{R}^{+} \times \mathbb{R}$, together with zero initial data

$$
D_{2}\left(0, x_{1}, x_{3}\right)=H_{1}\left(0, x_{1}, x_{3}\right)=H_{3}\left(0, x_{1}, x_{3}\right)=\chi\left(0, x_{1}, x_{3}\right)=0 \text { for } x_{1}>0, x_{3} \in \mathbb{R},
$$

and the impedance boundary condition

$$
H_{3}\left(t, 0, x_{3}\right)+a E_{2}\left(t, 0, x_{3}\right)=\varphi\left(t, x_{3}\right) \text { for } t>0, x_{3} \in \mathbb{R} .
$$

Recall that we have $\chi \geq 0$ and

$$
\partial_{1} H_{1}+\partial_{3} H_{3}=0
$$

We obtain the following global existence result.

Theorem 1 Let $\varphi \in H^{5}\left(\mathbb{R}^{2}\right)$, compactly supported in $\mathbb{R}^{+} \times \mathbb{R}$. Let $\varepsilon>0$. Then the smooth solution $W=\left(D_{2}, H_{1}, H_{3}, \chi\right)$ to the IBVP (2.16)-(2.17)-(2.18) is defined on $\left[0,+\infty\left[\left(T_{\varepsilon}^{*}=+\infty\right)\right.\right.$.

\section{Proof of Theorem 1.}

We fix $\varepsilon>0$ and we assume that $T_{\varepsilon}^{*}<+\infty$. Then from Proposition 2 , if $T_{\varepsilon}^{*}<+\infty$, then

$$
\lim _{T \rightarrow T_{\varepsilon}^{*}}\|W\|_{\mathcal{H}^{3}\left(\Omega_{T}\right)}=+\infty
$$

where

$$
\mathcal{H}^{p}\left(\Omega_{T}\right)=\left\{W \text { such that }\|W\|_{\mathcal{H}^{p}\left(\Omega_{T}\right)}:=\sum_{i=0}^{p}\left\|\partial_{t}^{i} W\right\|_{L^{\infty}\left(0, T ; H^{p-i}(\Omega)\right)}<+\infty\right\} .
$$


By variational estimates we will prove uniform bounds on $\|W\|_{\mathcal{H}^{3}\left(\Omega_{T}\right)}$ for $T<T_{\varepsilon}^{*}$ and so we will obtain a contradiction with $(2.20)$, which proves that $T_{\varepsilon}^{*}=+\infty$.

With this object, as in the 3 -d case, we extend the boundary condition $(2.20)$ : we replace $H_{3}\left(t, x_{1}, x_{3}\right)$ by $H_{3}\left(t, x_{1}, x_{3}\right)+\varphi\left(t, x_{3}\right) \eta\left(x_{1}\right)$ where $\eta$ is a smooth function compactly supported in $\mathbb{R}^{+}$, equal to 1 in a neighbourhood of 0 . Furthermore, to preserve the divergence free condition (2.19) we replace $H_{1}\left(t, x_{1}, x_{3}\right)$ by $H_{1}\left(t, x_{1}, x_{3}\right)+\partial_{3} \varphi\left(t, x_{3}\right) \int_{x_{1}}^{+\infty} \eta(s) d s$. Then the system (2.16)-(2.17)-(2.18) becomes, in the variable $V=\left(E_{2}, H_{1}, H_{3}, \chi\right)$ :

$$
\left\{\begin{array}{l}
(1+\chi) \partial_{t} E_{2}-\partial_{3} H_{1}+\partial_{1} H_{3}=-\partial_{t} \chi E_{2}+G_{1}, \\
\partial_{t} H_{1}-\partial_{3} E_{2}=G_{2} \\
\partial_{t} H_{3}+\partial_{1} E_{2}=G_{3} \\
\partial_{t} \chi=\frac{1}{\varepsilon}\left(\left|E_{2}\right|^{2}-\chi\right),
\end{array}\right.
$$

together with both zero initial data and homogeneous boundary condition:

$$
\begin{gathered}
V\left(0, x_{1}, x_{3}\right)=0, \\
H_{3}\left(t, 0, x_{3}\right)+a E_{2}\left(t, 0, x_{3}\right)=0,
\end{gathered}
$$

where

$$
\begin{aligned}
& G_{1}\left(t, x_{1}, x_{3}\right)=\partial_{3}^{2} \varphi\left(t, x_{3}\right) \int_{x_{1}}^{+\infty} \eta(s) d s-\varphi\left(t, x_{3}\right) \eta^{\prime}\left(x_{1}\right), \\
& G_{2}\left(t, x_{1}, x_{3}\right)=\partial_{t} \partial_{3} \varphi\left(t, x_{3}\right) \int_{x_{1}}^{+\infty} \eta(s) d s, \\
& G_{3}\left(t, x_{1}, x_{3}\right)=-\partial_{t} \varphi\left(t, x_{3}\right) \eta\left(x_{1}\right) .
\end{aligned}
$$

We recall that the field $H$ remains divergence free:

$$
\partial_{1} H_{1}+\partial_{3} H_{3}=0
$$

In this case the dissipation formulae (2.13)-(2.14) read

$$
\frac{1}{2} \frac{d}{d t} \int_{\Omega}\left((1+\chi)\left|E_{2}\right|^{2}+|H|^{2}+\frac{1}{2}|\chi|^{2}\right) d x_{1} d x_{3}+\frac{\varepsilon}{2} \int_{\Omega}\left|\partial_{t} \chi\right|^{2} d x+\int_{\Gamma} a\left|E_{2}\right|^{2} d x_{3}=\int_{\Omega} G \cdot U d x .
$$

and

$$
\begin{array}{r}
\frac{1}{2} \frac{d}{d t} \int_{\Omega}\left((1+\chi)\left|\partial_{t} E_{2}\right|^{2}+\left|\partial_{t} H\right|^{2}+\frac{1}{2}\left|\partial_{t} \chi\right|^{2}\right) d x_{1} d x_{3}+\frac{3}{2 \varepsilon} \int_{\Omega}\left|E_{2}\right|^{2}\left|\partial_{t} E_{2}\right|^{2} d x+\frac{\varepsilon}{2} \int_{\Omega}\left|\partial_{t t} \chi\right|^{2} d x \\
+\int_{\Gamma} a\left|\partial_{t} E_{2}\right|^{2} d x_{3}=\frac{3}{2 \varepsilon} \int_{\Omega} \chi\left|\partial_{t} E\right|^{2} d x+\int_{\Omega} \partial_{t} G \cdot \partial_{t} U d x
\end{array}
$$

where $G=\left(G_{1}, G_{2}, G_{3}\right)$ and $U=\left(E_{2}, H_{1}, H_{3}\right)$.

From (2.25), using Gronwall lemma (recall that $\chi \geq 0$ ), there exists a constant $C$ such that

$$
\|V\|_{\mathcal{H}^{0}\left(\Omega_{T_{\varepsilon}^{*}}\right)}=\|V\|_{L^{\infty}\left(0, T_{\varepsilon}^{*} ; L^{2}(\Omega)\right)} \leq C .
$$

Estimates on the first order time derivatives.

We recall the derivatives of (2.21)-(2.22)-(2.23) with respect to $t$ : 


$$
\left\{\begin{array}{c}
(1+\chi) \partial_{t}^{2} E_{2}+2 \partial_{t} \chi \partial_{t} E_{2}+\partial_{t}^{2} \chi E_{2}-\partial_{3} \partial_{t} H_{1}+\partial_{1} \partial_{t} H_{3}=\partial_{t} G_{1} \\
\partial_{t}^{2} H_{1}-\partial_{3} \partial_{t} E_{2}=\partial_{t} G_{2} \\
\partial_{t}^{2} H_{3}+\partial_{1} \partial_{t} E_{2}=\partial_{t} G_{3} \\
\partial_{t}^{2} \chi=\frac{1}{\varepsilon}\left(2 E_{2} \partial_{t} E_{2}-\partial_{t} \chi\right) \\
\partial_{t} H_{3}\left(t, 0, x_{3}\right)+a \partial_{t} E_{2}\left(t, 0, x_{3}\right)=0
\end{array}\right.
$$

By the equations, we obtain zero initial data

$$
\partial_{t} V\left(0, x_{1}, x_{3}\right)=0 .
$$

From these equations, we obtained (2.26), and by Gronwall lemma, there exists $C>0$ such that

$$
\left\|\partial_{t} V\right\|_{L^{\infty}\left(0, T_{\varepsilon}^{*} ; L^{2}(\Omega)\right)} \leq C .
$$

The last two equations in (2.21) yield

$$
\left\|E_{2}\right\|_{L^{\infty}\left(0, T_{\varepsilon}^{*} ; H^{1}(\Omega)\right)} \leq C .
$$

Solving (2.22) we get

$$
\chi(t, x)=\frac{1}{\varepsilon} \int_{0}^{t} \exp \left(\frac{s-t}{\varepsilon}\right)\left|E_{2}(s, x)\right|^{2} d s .
$$

From Sobolev theorem and (2.32), for all $p \in[2,+\infty[$ there exists $C>0$ such that

$$
\left\|E_{2}\right\|_{L^{\infty}\left(0, T_{\varepsilon}^{*} ; L^{p}(\Omega)\right)} \leq C,
$$

and so with (2.33), for all $p$ with $1 \leq p<+\infty$, there exists $C$ such that

$$
\|\chi\|_{L^{\infty}\left(0, T_{\varepsilon}^{*} ; L^{p}(\Omega)\right)} \leq C .
$$

So by $(2.22)$ we obtain that, for all $p \in[1,+\infty[$,

$$
\left\|\partial_{t} \chi\right\|_{L^{\infty}\left(0, T_{\varepsilon}^{*} ; L^{p}(\Omega)\right)} \leq C .
$$

\section{Estimates on the second order time derivatives.}

We differentiate (2.28)-(2.29)-(2.30) with respect to $t$. We obtain

$$
\left\{\begin{array}{l}
(1+\chi) \partial_{t}^{3} E_{2}+3 \partial_{t} \chi \partial_{t}^{2} E_{2}+3 \partial_{t}^{2} \chi \partial_{t} E_{2}+\partial_{t}^{3} \chi E_{2}-\partial_{3} \partial_{t}^{2} H_{1}+\partial_{1} \partial_{t}^{2} H_{3}=\partial_{t}^{2} G_{1}, \\
\partial_{t}^{3} H_{1}-\partial_{3} \partial_{t}^{2} E_{2}=\partial_{t}^{2} G_{2} \\
\partial_{t}^{3} H_{3}+\partial_{1} \partial_{t}^{2} E_{2}=\partial_{t}^{2} G_{3} \\
\partial_{t}^{3} \chi=\frac{1}{\varepsilon}\left(2\left|\partial_{t} E_{2}\right|^{2}+2 E_{2} \partial_{t}^{2} E_{2}-\partial_{t}^{2} \chi\right)
\end{array}\right.
$$

with homogeneous boundary condition

$$
\partial_{t}^{2} H_{3}\left(t, 0, x_{3}\right)+a \partial_{t}^{2} E_{2}\left(t, 0, x_{3}\right)=0,
$$

and by the equations, the initial data vanish

$$
\partial_{t}^{2} V\left(0, x_{1}, x_{3}\right)=0 .
$$


Taking the inner product of (2.37) with $\partial_{t}^{2} U$ we obtain

$$
\begin{aligned}
& \frac{1}{2} \frac{d}{d t} \int_{\Omega}\left((1+\chi)\left|\partial_{t}^{2} E_{2}\right|^{2}+\left|\partial_{t}^{2} H\right|^{2}\right) d x_{1} d x_{3}+\int_{\Gamma} a\left|\partial_{t}^{2} E_{2}\right|^{2} d x_{3} \\
& +\frac{5}{2} \int_{\Omega} \partial_{t} \chi\left|\partial_{t}^{2} E_{2}\right|^{2} d x+3 \int_{\Omega} \partial_{t}^{2} \chi \partial_{t} E_{2} \partial_{t}^{2} E_{2}+\int_{\Omega} \partial_{t}^{3} \chi E_{2} \partial_{t}^{2} E_{2}=\int_{\Omega} \partial_{t}^{2} G \cdot \partial_{t}^{2} U d x
\end{aligned}
$$

Equation (2.22) yields

$$
\int_{\Omega} \partial_{t} \chi\left|\partial_{t}^{2} E_{2}\right|^{2} d x=\frac{1}{\varepsilon} \int_{\Omega}\left|E_{2}\right|^{2}\left|\partial_{t}^{2} E_{2}\right|^{2} d x-\frac{1}{\varepsilon} \int_{\Omega} \chi\left|\partial_{t}^{2} E_{2}\right|^{2} d x .
$$

From (2.29) we get

$$
\int_{\Omega} \partial_{t}^{2} \chi \partial_{t} E_{2} \partial_{t}^{2} E_{2} d x=\frac{2}{\varepsilon} \int_{\Omega} E_{2}\left|\partial_{t} E_{2}\right|^{2} \partial_{t}^{2} E_{2} d x-\frac{1}{\varepsilon} \int_{\Omega} \partial_{t} \chi \partial_{t} E_{2} \partial_{t}^{2} E_{2} d x .
$$

From (2.38) we have

$$
\begin{array}{r}
\int_{\Omega} \partial_{t}^{3} \chi E_{2} \partial_{t}^{2} E_{2} d x=\frac{2}{\varepsilon} \int_{\Omega} E_{2}\left|\partial_{t} E_{2}\right|^{2} \partial_{t}^{2} E_{2} d x+\frac{2}{\varepsilon} \int_{\Omega}\left|E_{2}\right|^{2}\left|\partial_{t}^{2} E_{2}\right|^{2} d x-\frac{2}{\varepsilon^{2}} \int_{\Omega}\left|E_{2}\right|^{2} \partial_{t} E_{2} \partial_{t}^{2} E_{2} d x \\
+\frac{1}{\varepsilon^{2}} \int_{\Omega} \partial_{t} \chi E_{2} \partial_{t}^{2} E_{2} d x .
\end{array}
$$

So we obtain

$$
\begin{aligned}
& \frac{1}{2} \frac{d}{d t} \int_{\Omega}\left((1+\chi)\left|\partial_{t}^{2} E_{2}\right|^{2}+\left|\partial_{t}^{2} H\right|^{2}\right) d x_{1} d x_{3}+\int_{\Gamma} a\left|\partial_{t}^{2} E_{2}\right|^{2} d x_{3}+\frac{9}{2 \varepsilon} \int_{\Omega}\left|E_{2}\right|^{2}\left|\partial_{t}^{2} E_{2}\right|^{2} d x= \\
& \int_{\Omega} \partial_{t}^{2} G \cdot \partial_{t}^{2} U d x+\frac{5}{2 \varepsilon} \int_{\Omega} \chi\left|\partial_{t}^{2} E_{2}\right|^{2} d x-\frac{8}{\varepsilon} \int_{\Omega} E_{2} \partial_{t}^{2} E_{2}\left|\partial_{t} E_{2}\right|^{2} d x+\frac{3}{\varepsilon} \int_{\Omega} \partial_{t} \chi \partial_{t} E_{2} \partial_{t}^{2} E_{2} d x \\
&+\frac{2}{\varepsilon^{2}} \int_{\Omega}\left|E_{2}\right|^{2} \partial_{t} E_{2} \partial_{t}^{2} E_{2} d x-\frac{1}{\varepsilon^{2}} \int_{\Omega} \partial_{t} \chi E_{2} \partial_{t}^{2} E_{2} d x
\end{aligned}
$$

By (2.28) and (2.31) we have

$$
\left\|\partial_{t} E_{2}\right\|_{\left.H^{1}(\Omega)\right)} \leq C\left(1+\left\|\partial_{t}^{2} H\right\|_{L^{2}(\Omega)}\right)
$$

Let us estimate the right hand side terms in (2.40). First we have

$$
\left.\left.\left|\frac{8}{\varepsilon} \int_{\Omega} E_{2} \partial_{t}^{2} E_{2}\right| \partial_{t} E_{2}\right|^{2} d x\left|\leq \frac{9}{4 \varepsilon} \int_{\Omega}\right| E_{2}\right|^{2}\left|\partial_{t}^{2} E_{2}\right|^{2} d x+K(\varepsilon) \int_{\Omega}\left|\partial_{t} E_{2}\right|^{4} d x .
$$

By interpolation inequalities and Sobolev theorem, we have

$$
\|u\|_{L^{4}(\Omega)} \leq C\|u\|_{H^{\frac{1}{2}(\Omega)}} \leq\|u\|_{L^{2}(\Omega)}^{\frac{1}{2}}\|u\|_{H^{1}(\Omega)}^{\frac{1}{2}} .
$$

So using (2.41)

$$
\left.\left.\left|\frac{8}{\varepsilon} \int_{\Omega} E_{2} \partial_{t}^{2} E_{2}\right| \partial_{t} E_{2}\right|^{2} d x\left|\leq \frac{9}{4 \varepsilon} \int_{\Omega}\right| E_{2}\right|^{2}\left|\partial_{t}^{2} E_{2}\right|^{2} d x+K(\varepsilon)\left(1+\left\|\partial_{t}^{2} H\right\|_{L^{2}(\Omega)}^{2}\right) .
$$

By (2.36) and (2.41) we obtain

$$
\begin{aligned}
\left|\int_{\Omega} \partial_{t} \chi \partial_{t} E_{2} \partial_{t}^{2} E_{2} d x\right| & \leq C\left\|\partial_{t} \chi\right\|_{L^{4}(\Omega)}\left\|\partial_{t} E_{2}\right\|_{L^{4}(\Omega)}\left\|\partial_{t}^{2} E_{2}\right\|_{L^{2}(\Omega)} \\
& \leq C\left\|\partial_{t} E_{2}\right\|_{H^{1}(\Omega)}\left\|\partial_{t}^{2} E_{2}\right\|_{L^{2}(\Omega)} \\
& \leq C\left(1+\left\|\partial_{t}^{2} U\right\|_{L^{2}(\Omega)}^{2}\right)
\end{aligned}
$$


In the same way, (2.34) and (2.41) yield

$$
\left.\left|\int_{\Omega}\right| E_{2}\right|^{2} \partial_{t} E_{2} \partial_{t}^{2} E_{2} d x \mid \leq C\left(1+\left\|\partial_{t}^{2} U\right\|_{L^{2}(\Omega)}^{2}\right)
$$

and by (2.34) and (2.36) we have

$$
\left|\int_{\Omega} \partial_{t} \chi E_{2} \partial_{t}^{2} E_{2} d x\right| \leq C\left(1+\left\|\partial_{t}^{2} U\right\|_{L^{2}(\Omega)}^{2}\right) .
$$

Using (2.42), (2.43), (2.44), (2.45) in (2.40), we obtain

$$
\frac{1}{2} \frac{d}{d t} \int_{\Omega}\left((1+\chi)\left|\partial_{t}^{2} E_{2}\right|^{2}+\left|\partial_{t}^{2} H\right|^{2}\right) d x_{1} d x_{3} \leq C\left(1+\left\|\partial_{t}^{2} U\right\|_{L^{2}(\Omega)}^{2}\right)+\frac{5}{2 \varepsilon} \int_{\Omega} \chi\left|\partial_{t}^{2} E_{2}\right|^{2} d x,
$$

so by Gronwall lemma there exists $C=C\left(T_{\varepsilon}^{*}\right)$ such that

$$
\left\|\partial_{t}^{2} U\right\|_{L^{\infty}\left(0, T_{\varepsilon}^{*} ; L^{2}(\Omega)\right)} \leq C .
$$

So by $(2.41)$

$$
\left\|\partial_{t} E_{2}\right\|_{L^{\infty}\left(0, T_{\varepsilon}^{*} ; H^{1}(\Omega)\right)} \leq C,
$$

and by Sobolev theorem, for all $p, 2 \leq p<+\infty$,

$$
\left\|\partial_{t} E_{2}\right\|_{L^{\infty}\left(0, T_{\varepsilon}^{*} ; L^{p}(\Omega)\right)} \leq C .
$$

As $\partial_{t}^{2} \chi=\frac{2}{\varepsilon} E_{2} \partial_{t} E_{2}-\frac{1}{\varepsilon} \partial_{t} \chi$, by (2.34), (2.36), (2.48) we have for $1 \leq p<+\infty$ :

$$
\left\|\partial_{t}^{2} \chi\right\|_{L^{\infty}\left(0, T_{\varepsilon}^{*} ; L^{p}(\Omega)\right)} \leq C .
$$

Estimates on the third order time derivatives.

We differentiate (2.37)-(2.38)-(2.39) with respect to $t$ :

$$
\left\{\begin{array}{l}
(1+\chi) \partial_{t}^{4} E_{2}+4 \partial_{t} \chi \partial_{t}^{3} E_{2}+6 \partial_{t}^{2} \chi \partial_{t}^{2} E_{2}+4 \partial_{t}^{3} \chi \partial_{t} E_{2}+\partial_{t}^{4} \chi E_{2}-\partial_{3} \partial_{t}^{3} H_{1}+\partial_{1} \partial_{t}^{3} H_{3}=\partial_{t}^{3} G_{1}, \\
\partial_{t}^{4} H_{1}-\partial_{3} \partial_{t}^{3} E_{2}=\partial_{t}^{3} G_{2}, \\
\partial_{t}^{4} H_{3}+\partial_{1} \partial_{t}^{3} E_{2}=\partial_{t}^{3} G_{3}, \\
\partial_{t}^{4} \chi=\frac{6}{\varepsilon} \partial_{t} E_{2} \partial_{t}^{2} E_{2}+\frac{2}{\varepsilon} E_{2} \partial_{t}^{3} E_{2}-\frac{2}{\varepsilon^{2}}\left|\partial_{t} E_{2}\right|^{2}-\frac{2}{\varepsilon^{2}} E_{2} \partial_{t}^{2} E_{2}+\frac{1}{\varepsilon^{2}} \partial_{t}^{2} \chi,
\end{array}\right.
$$

together with homogeneous boundary condition:

$$
\partial_{t}^{3} H_{3}\left(t, 0, x_{3}\right)+a \partial_{t}^{3} E_{2}\left(t, 0, x_{3}\right)=0,
$$

and by the equations, the initial data vanish:

$$
\partial_{t}^{3} V\left(0, x_{1}, x_{3}\right)=0 .
$$

We take the inner product of (2.50) with $\partial_{t}^{3} U$ and we obtain

$$
\begin{aligned}
& \frac{1}{2} \frac{d}{d t} \int_{\Omega}\left((1+\chi)\left|\partial_{t}^{3} E_{2}\right|^{2}+\left|\partial_{t}^{3} H\right|^{2}\right) d x_{1} d x_{3}+\int_{\Gamma} a\left|\partial_{t}^{3} E_{2}\right|^{2} d x_{3}+\frac{7}{2} \int_{\Omega} \partial_{t} \chi\left|\partial_{t}^{3} E_{2}\right|^{2} d x+6 \int_{\Omega} \partial_{t}^{2} \chi \partial_{t}^{2} E_{2} \partial_{t}^{3} E_{2} \\
& +4 \int_{\Omega} \partial_{t}^{3} \chi \partial_{t} E_{2} \partial_{t}^{3} E_{2}+\int_{\Omega} \partial_{t}^{4} \chi E_{2} \partial_{t}^{3} E_{2}=\int_{\Omega} \partial_{t}^{3} G \cdot \partial_{t}^{3} U d x .
\end{aligned}
$$


Equation (2.22) yields

$$
\int_{\Omega} \partial_{t} \chi\left|\partial_{t}^{3} E_{2}\right|^{2} d x=\frac{1}{\varepsilon} \int_{\Omega}\left|E_{2}\right|^{2}\left|\partial_{t}^{3} E_{2}\right|^{2} d x-\frac{1}{\varepsilon} \int_{\Omega} \chi\left|\partial_{t}^{3} E_{2}\right|^{2} d x
$$

From (2.38)

$$
\int_{\Omega} \partial_{t}^{3} \chi \partial_{t} E_{2} \partial_{t}^{3} E_{2}=\frac{2}{\varepsilon} \int_{\Omega}\left|\partial_{t} E_{2}\right|^{3} \partial_{t}^{3} E_{2} d x+\frac{2}{\varepsilon} \int_{\Omega} E_{2} \partial_{t} E_{2} \partial_{t}^{2} E_{2} \partial_{t}^{3} E_{2} d x-\int_{\Omega} \partial_{t}^{2} \chi \partial_{t} E_{2} \partial_{t}^{3} E_{2} .
$$

From (2.51), we get

$$
\begin{aligned}
\int_{\Omega} \partial_{t}^{4} \chi E_{2} \partial_{t}^{3} E_{2}=\frac{6}{\varepsilon} \int_{\Omega} E_{2} \partial_{t} E_{2} \partial_{t}^{2} E_{2} \partial_{t}^{3} E_{2} d x+ & \frac{2}{\varepsilon} \int_{\Omega}\left|E_{2}\right|^{2}\left|\partial_{t}^{3} E_{2}\right|^{2} d x-\frac{2}{\varepsilon^{2}} \int_{\Omega} E_{2}\left|\partial_{t} E_{2}\right|^{2} \partial_{t}^{3} E_{2} d x \\
& -\frac{2}{\varepsilon^{2}} \int_{\Omega}\left|E_{2}\right|^{2} \partial_{t}^{2} E_{2} \partial_{t}^{3} E_{2} d x+\frac{1}{\varepsilon^{2}} \int_{\Omega} \partial_{t}^{2} \chi E_{2} \partial_{t}^{3} E_{2} .
\end{aligned}
$$

So we arrive at

$$
\begin{array}{r}
\frac{1}{2} \frac{d}{d t} \int_{\Omega}\left((1+\chi)\left|\partial_{t}^{3} E_{2}\right|^{2}+\left|\partial_{t}^{3} H\right|^{2}\right) \\
d x_{1} d x_{3}+\int_{\Gamma} a\left|\partial_{t}^{3} E_{2}\right|^{2} d x_{3}+\frac{11}{2 \varepsilon} \int_{\Omega}\left|E_{2}\right|^{2}\left|\partial_{t}^{3} E_{2}\right|^{2} d x \\
=\int_{\Omega} \partial_{t}^{3} G \cdot \partial_{t}^{3} U d x+\frac{7}{2 \varepsilon} \int_{\Omega} \chi\left|\partial_{t}^{3} E_{2}\right|^{2} d x+I_{1}+I_{2},
\end{array}
$$

where

$$
\begin{aligned}
I_{1}= & -6 \int_{\Omega} \partial_{t}^{2} \chi \partial_{t}^{2} E_{2} \partial_{t}^{3} E_{2} d x-\frac{14}{\varepsilon} \int_{\Omega} E_{2} \partial_{t} E_{2} \partial_{t}^{2} E_{2} \partial_{t}^{3} E_{2} d x+\frac{2}{\varepsilon} \int_{\Omega}\left|E_{2}\right|^{2} \partial_{t}^{2} E_{2} \partial_{t}^{3} E_{2} d x, \\
I_{2}= & -\frac{8}{\varepsilon} \int_{\Omega}\left|\partial_{t} E_{2}\right|^{3} \partial_{t}^{3} E_{2} d x+\frac{4}{\varepsilon} \int_{\Omega} \partial_{t}^{2} \chi \partial_{t} E_{2} \partial_{t}^{3} E_{2} d x+\frac{2}{\varepsilon^{2}} \int_{\Omega} E_{2}\left|\partial_{t} E_{2}\right|^{2} \partial_{t}^{3} E_{2} d x \\
& -\frac{1}{\varepsilon^{2}} \int_{\Omega} \partial_{t}^{2} \chi E_{2} \partial_{t}^{3} E_{2} d x .
\end{aligned}
$$

From (2.37) and (2.46) we have

$$
\left\|\partial_{t}^{2} E_{2}\right\|_{H^{1}(\Omega)} \leq K\left(1+\left\|\partial_{t}^{3} H\right\|_{L^{2}(\Omega)}\right) .
$$

So by Sobolev theorem, (2.34), (2.48), (2.49) and (2.53), we get

$$
\left|I_{1}\right| \leq C\left(1+\left\|\partial_{t}^{3} U\right\|_{L^{2}(\Omega)}^{2}\right) .
$$

By (2.34), (2.48), (2.49), we have

$$
\left|I_{2}\right| \leq C\left\|\partial_{t}^{3} E_{2}\right\|_{L^{2}(\Omega)} .
$$

Estimates (2.54) and (2.55) together with (2.52) imply

$$
\frac{1}{2} \frac{d}{d t} \int_{\Omega}\left((1+\chi)\left|\partial_{t}^{3} E_{2}\right|^{2}+\left|\partial_{t}^{3} H\right|^{2}\right) d x_{1} d x_{3} \leq C\left(1+\left\|\partial_{t}^{3} U\right\|_{L^{2}(\Omega)}^{2}\right)+\frac{7}{2 \varepsilon} \int_{\Omega} \chi\left|\partial_{t}^{3} E_{2}\right|^{2} d x .
$$

So there exists a constant $C=C\left(T_{\varepsilon}^{*}\right)>0$ such that

$$
\left\|\partial_{t}^{3} U\right\|_{L^{\infty}\left(0, T_{\varepsilon}^{*} ; L^{2}(\Omega)\right)} \leq C .
$$

By (2.53)

$$
\left\|\partial_{t}^{2} E_{2}\right\|_{L^{\infty}\left(0, T_{\varepsilon}^{*} ; H^{1}(\Omega)\right)} \leq C,
$$


and, for all $p, 2 \leq p<+\infty$,

$$
\left\|\partial_{t}^{2} E_{2}\right\|_{L^{\infty}\left(0, T_{\varepsilon}^{*} ; L^{p}(\Omega)\right)} \leq C .
$$

As $\partial_{t}^{3} \chi=\frac{2}{\varepsilon}\left|\partial_{t} E_{2}\right|^{2}+\frac{2}{\varepsilon} E_{2} \partial_{t}^{2} E_{2}-\frac{1}{\varepsilon} \partial_{t}^{2} \chi$, using (2.34), (2.48), (2.49) and (2.56), for $1 \leq p<+\infty$,

$$
\left\|\partial_{t}^{3} \chi\right\|_{L^{\infty}\left(0, T_{\varepsilon}^{*} ; L^{p}(\Omega)\right)} \leq C .
$$

Estimates in $\mathcal{H}^{3}\left(\Omega_{T_{\varepsilon}^{*}}\right)$.

In order to estimate the space derivatives of $H$ we recall the following div-curl lemma (see [9]).

Lemma 1 Let $\Omega=\left\{\left(x_{1}, x_{3}\right), x_{1}>0\right\}$. We denote $\Gamma=\partial \Omega$. Let $H=\left(H_{1}, H_{3}\right) \in H^{k}(\Omega)$ such that $\operatorname{div} H=\partial_{1} H_{1}+\partial_{3} H_{3} \in H^{k}(\Omega)$, curl $H=-\partial_{1} H_{3}+\partial_{3} H_{1} \in H^{k}(\Omega), H_{3} \in H^{k+\frac{1}{2}}(\Gamma)$. Then $H \in H^{k+1}(\Omega)$ and we have

$$
\|H\|_{H^{k+1}(\Omega)} \leq C\left(\|H\|_{H^{k}(\Omega)}+\|\operatorname{div} H\|_{H^{k}(\Omega)}+\|\operatorname{curl} H\|_{H^{k}(\Omega)}+\|H\|_{H^{k+\frac{1}{2}(\Gamma)}}\right) .
$$

By the first equation in (2.21), using (2.34), (2.35), (2.36) and (2.48), we obtain

$$
\|\operatorname{curl} H\|_{L^{\infty}\left(0, T_{\varepsilon}^{*} ; L^{2}(\Omega)\right)} \leq C .
$$

Recall that $\operatorname{div} H=0$ and on the boundary $\Gamma, H_{3}\left(t, 0, x_{3}\right)=-a E_{2}\left(t, 0, x_{3}\right)$, so by $(2.32)$,

$$
\left\|H_{3}(t, 0, .)\right\|_{L^{\infty}\left(0, T_{\varepsilon}^{*} ; H^{\frac{1}{2}}(\Gamma)\right)} \leq C .
$$

Using lemma 1, we obtain

$$
\|H\|_{L^{\infty}\left(0, T_{\varepsilon}^{*} ; H^{1}(\Omega)\right)} \leq C .
$$

By the first equation in (2.28), using (2.34), (2.35), (2.36), (2.48), (2.49) and (2.56), we get

$$
\left\|\operatorname{curl} \partial_{t} H\right\|_{L^{\infty}\left(0, T_{\varepsilon}^{*} ; L^{2}(\Omega)\right)} \leq C .
$$

Condition (2.23) together with (2.47) yield

$$
\left\|\partial_{t} H_{3}(t, 0, .)\right\|_{L^{\infty}\left(0, T_{\varepsilon}^{*} ; H^{\frac{1}{2}}(\Gamma)\right)} \leq C,
$$

thus

$$
\left\|\partial_{t} H\right\|_{L^{\infty}\left(0, T_{\varepsilon}^{*} ; H^{1}(\Omega)\right)} \leq C .
$$

By the second and the third equations in (2.21), by (2.32) and (2.57),

$$
\left\|E_{2}\right\|_{L^{\infty}\left(0, T_{\varepsilon}^{*} ; H^{2}(\Omega)\right)} \leq C
$$

and by Sobolev theorem,

$$
\left\|E_{2}\right\|_{L^{\infty}\left(\left[0, T_{\varepsilon}^{*}[\times \Omega)\right.\right.} \leq C .
$$

As $H^{2}(\Omega)$ is an algebra, using (2.33), we obtain

$$
\|\chi\|_{L^{\infty}\left(0, T_{\varepsilon}^{*} ; H^{2}(\Omega)\right)} \leq C,
$$

and by Sobolev theorem,

$$
\|\chi\|_{L^{\infty}\left(\left[0, T_{\varepsilon}^{*}[\times \Omega)\right.\right.} \leq C .
$$

Since $\partial_{t} \chi=\frac{1}{\varepsilon}\left(\left|E_{2}\right|^{2}-\chi\right)$,

$$
\left\|\partial_{t} \chi\right\|_{L^{\infty}\left(0, T_{\varepsilon}^{*} ; H^{2}(\Omega)\right)} \leq C,
$$

and by Sobolev theorem,

$$
\left\|\partial_{t} \chi\right\|_{L^{\infty}\left(\left[0, T_{\varepsilon}^{*}[\times \Omega)\right.\right.} \leq C .
$$


In addition with (2.38) we have

$$
\left\|\partial_{t}^{2} \chi\right\|_{L^{\infty}\left(0, T_{\varepsilon}^{*} ; H^{1}(\Omega)\right)} \leq C .
$$

By the first equation in (2.21), using (2.47), (2.58), (2.59), (2.60), we obtain

$$
\|\operatorname{curl} H\|_{L^{\infty}\left(0, T_{\varepsilon}^{*} ; H^{1}(\Omega)\right)} \leq C .
$$

Using (2.58), (2.23) yields

$$
\left\|H_{3}(t, 0, .)\right\|_{L^{\infty}\left(0, T_{\varepsilon}^{*} ; H^{\frac{3}{2}}(\Gamma)\right)} \leq C
$$

so by Lemma 1 ,

$$
\|H\|_{L^{\infty}\left(0, T_{\varepsilon}^{*} ; H^{2}(\Omega)\right)} \leq C .
$$

By analogous arguments, we prove successively that

$$
\begin{gathered}
\left\|\partial_{t}^{2} H\right\|_{L^{\infty}\left(0, T_{\varepsilon}^{*} ; H^{1}(\Omega)\right)} \leq C, \\
\left\|\partial_{t} E_{2}\right\|_{L^{\infty}\left(0, T_{\varepsilon}^{*} ; H^{2}(\Omega)\right)} \leq C, \\
\left\|\partial_{t}^{2} \chi\right\|_{L^{\infty}\left(0, T_{\varepsilon}^{*} ; H^{2}(\Omega)\right)} \leq C, \\
\left\|\partial_{t} H\right\|_{L^{\infty}\left(0, T_{\varepsilon}^{*} ; H^{2}(\Omega)\right)} \leq C, \\
\left\|E_{2}\right\|_{L^{\infty}\left(0, T_{\varepsilon}^{*} ; H^{3}(\Omega)\right)} \leq C, \\
\|\chi\|_{L^{\infty}\left(0, T_{\varepsilon}^{*} ; H^{3}(\Omega)\right)} \leq C, \\
\|H\|_{L^{\infty}\left(0, T_{\varepsilon}^{*} ; H^{3}(\Omega)\right)} \leq C .
\end{gathered}
$$

Finally,

$$
\|V\|_{\mathcal{H}^{3}\left(\Omega_{T_{\varepsilon}^{*}}\right)} \leq C .
$$

This estimate contradicts $(2.20)$, so $T_{\varepsilon}^{*}=+\infty$. The proof of Theorem 1 is complete.

\subsection{Global existence for the 1-d Kerr-Debye system.}

The one-dimensional Kerr Debye IBVP writes:

$$
\left\{\begin{array}{l}
\partial_{t} d+\partial_{x} h=0, \\
\partial_{t} h+\partial_{x} e=0, \\
\partial_{t} \chi=\frac{1}{\varepsilon}\left(e^{2}-\chi\right), \\
d=(1+\chi) e
\end{array}\right.
$$

for $(t, x) \in \mathbb{R}^{+} \times \mathbb{R}^{+}$, together with both zero initial data

$$
(d, h, \chi)(0, x)=0 \text { for } x \geq 0
$$

and the boundary condition

$$
h(t, 0)+a e(t, 0=\varphi(t) \text { for } t \geq 0 .
$$

Theorem 2 Let $\varphi \in H^{4}(\mathbb{R})$ compactly supported in $\mathbb{R}^{+}$. Let $\varepsilon>0$. Then the smooth solution $(d, h, \chi)$ to the IBVP (2.61)-(2.62)-(2.63) is defined on $[0,+\infty[$. 
The proof of Theorem 2 is analogous to the proof of Theorem 1. The one-dimensional case is easier: the divergence free condition is irrelevant and the space regularity is obtained directly by the equations. In addition, by Sobolev theorem, the $H^{1}$-estimates implies bounds in the $L^{\infty}$-norm. We refer to [7] for the detailed proof of the global existence for the Cauchy problem.

Remark 3 Using the general stability results in [12] and [1], we can observe that for the 1-d Kerr-Debye model, a constant equilibrium state $\left(\bar{e}, \bar{h}, \bar{\chi}=(\bar{e})^{2}\right)$ is stable if $\bar{e} \neq 0$. The stability property if $\bar{e}=0$ in the one-dimensional case and in the two-dimensional TE case remains an open problem.

Remark 4 It seems more difficult to obtain the global existence for both the 2-dTM and the 3-d models. First we lack for an adapted div-curl lemma to obtain the space regularity. Indeed, in both cases, the divergence free condition (2.7) is nonlinear in $(\chi, E)$. In addition, the Sobolev embeddings used in the 2-d case don't work in the 3-d case.

\section{Convergence result.}

First we replace the Kerr-Debye model in the general framework of [8]. The equilibrium manifold in (2.4) is defined by:

$$
\mathcal{V}=\left\{(D, H, \chi) \in \mathbb{R}^{7}, \chi=|E|^{2}=(1+\chi)^{-2}|D|^{2}\right\},
$$

so that the reduced system associated with the Kerr-Debye model (2.4) is the Kerr model (2.1).

The strictly convex entropy $\mathcal{E}_{K D}$ satisfies the stability condition in the definition 2.1 of [8] and, on the equilibrium manifold $\mathcal{V}$, we have the relation:

$$
\mathcal{E}_{K}(D, H)=\mathcal{E}_{K D}(D, H, \chi(D)) .
$$

Furthermore characteristic speeds associated to (2.4) and (2.1) are interlaced on the equilibrium manifold $\mathcal{V}$. In our case, by the previous calculations, in the 3 -d model we have

$$
\mu_{1}\left(|E|^{2}, \xi\right)=\lambda_{1}(E, \xi)=\mu_{2}\left(|E|^{2}, \xi\right) \leq \lambda_{2}(E, \xi) \leq 0=\mu_{3}=\lambda_{3}=\mu_{4}=\lambda_{4}=\mu_{5} .
$$

So the Kerr-Debye model is a relaxation approximation of the Kerr model, with the stability properties. In this context, it is natural to study the convergence of the solutions to the Kerr-Debye model as the relaxation coefficient tends to zero. The Cauchy problem is studied in [11]. Here we establish the convergence for the impedance IBVP.

\subsection{Entropic variables and main result.}

In order to prove the convergence result it is more convenient to use the entropic variables which are introduced in [12]. These variables are obtained taking the gradient of the convex entropy (2.8):

$$
\left\{\begin{array}{l}
\partial_{D} \mathcal{E}_{K D}=(1+\chi)^{-1} D=E, \\
\partial_{H} \mathcal{E}_{K D}=H, \\
\partial_{\chi} \mathcal{E}_{K D}=\frac{1}{2}\left(\chi-|E|^{2}\right):=v,
\end{array}\right.
$$

The IBVP (2.4)-(2.5)-(2.6) becomes (for $(t, x) \in \mathbb{R}^{+} \times \Omega$ ):

$$
A_{0}\left(W_{\varepsilon}\right) \partial_{t} W_{\varepsilon}+\sum_{j=1}^{3} A_{j} \partial_{j} W_{\varepsilon}=\frac{1}{\varepsilon} Q\left(W_{\varepsilon}\right),
$$

where 
- $W_{\varepsilon}=\left(\begin{array}{c}E_{\varepsilon} \\ H_{\varepsilon} \\ v_{\varepsilon}\end{array}\right), \quad Q\left(W_{\varepsilon}\right)=\left(\begin{array}{c}0 \\ 0 \\ -2 v_{\varepsilon}\end{array}\right)$,

- $A_{0}\left(W_{\varepsilon}\right)=\left(\begin{array}{ccc}\left(\left|E_{\varepsilon}\right|^{2}+2 v_{\varepsilon}+1\right) I_{3}+2 E_{\varepsilon}{ }^{t} E_{\varepsilon} & 0 & 2 E_{\varepsilon} \\ 0 & I_{3} & 0 \\ 2{ }^{t} E_{\varepsilon} & 0 & 2\end{array}\right)$,

- $\sum_{j=1}^{3} A_{j} \partial_{j}=\left(\begin{array}{ccc}0 & - \text { curl } & 0 \\ \text { curl } & 0 & 0 \\ 0 & 0 & 0\end{array}\right)$,

with the initial data

$$
E_{\varepsilon}(0, x)=H_{\varepsilon}(0, x)=0, v_{\varepsilon}(0, x)=0 \text { for } x \in \Omega,
$$

and with the boundary condition

$$
H_{\varepsilon} \times n+a\left(\left(E_{\varepsilon} \times n\right) \times n\right)=\varphi \text { for }(t, x) \in \mathbb{R}^{+} \times \Gamma .
$$

We observe that in the entropic variables $(E, H, v)$, the boundary condition is linear. In addition, with these variables, the equilibrium manifold is flat since $\mathcal{V}=\left\{(E, H, v) \in \mathbb{R}^{7}, v=0\right\}$ and the relaxation term is linear.

We rewrite the divergence free condition in the entropic variables:

$$
\operatorname{div}\left(\left(1+\left|E_{\varepsilon}\right|^{2}+2 v_{\varepsilon}\right) E_{\varepsilon}\right)=\operatorname{div} H_{\varepsilon}=0 .
$$

In the same way we can write the Kerr model in its entropic variables :

$$
\left\{\begin{array}{l}
\partial_{D} \mathcal{E}_{K}=E \\
\partial_{H} \mathcal{E}_{K}=H
\end{array}\right.
$$

The IBVP (2.1)-(2.2)-(2.3) becomes

$$
\left\{\begin{array}{l}
\left(\left(1+\left|E_{0}\right|^{2}\right) I_{3}+2 E_{0}{ }^{t} E_{0}\right) \partial_{t} E_{0}-\operatorname{curl} H_{0}=0, \\
\partial_{t} H_{0}+\operatorname{curl} H_{0}=0,
\end{array}\right.
$$

for $(t, x) \in \mathbb{R}^{+} \times \Omega$ together with zero initial data

$$
E_{0}(0, x)=H_{0}(0, x)=0 \text { for } x \in \Omega,
$$

and with the impedance boundary condition

$$
H_{0} \times n+a\left(\left(E_{0} \times n\right) \times n\right)=\varphi \text { for }(t, x) \in \mathbb{R}^{+} \times \Gamma .
$$

We remark that the divergence free condition is satisfied

$$
\operatorname{div}\left(\left(1+\left|E_{0}\right|^{2}\right) E_{0}\right)=\operatorname{div} H_{0}=0 .
$$

In these entropic variables the IBVP (3.5)-(3.6)-(3.7) is the reduced system of the IBVP (3.1)-(3.2)-(3.3). Let us recall the assumptions on the source term $\varphi$ in both problems:

$$
\begin{aligned}
& \varphi \text { is compactly supported in } \mathbb{R}^{+} \times \Gamma, \\
& \varphi \in H^{s}(\mathbb{R} \times \Gamma), s \geq 5 .
\end{aligned}
$$


Thus we can apply Proposition 1 and Proposition 2 with the source term $\varphi$ to define the solution $\left(E_{0}, H_{0}\right)$ to (3.5)-(3.6)-(3.7) on $\left[0, T^{*}\left[\right.\right.$ and the solution $\left(E_{\varepsilon}, H_{\varepsilon}, v_{\varepsilon}\right)$ to (3.1)-(3.2)-(3.3) on $\left[0, T_{\varepsilon}^{*}[\right.$. In addition we choose $s$ great enough to ensure a sufficient regularity on the profile $\left(E_{0}, H_{0}\right)$ (see Remark 1$)$.

As in the previous section we introduce for $T>0$ and $k \in \mathbb{N}$ :

$$
\mathcal{H}^{k}\left(\Omega_{T}\right)=\left\{W \text { such that }\|W\|_{\mathcal{H}^{k}\left(\Omega_{T}\right)}:=\sum_{i=0}^{k}\left\|\partial_{t}^{i} W\right\|_{L^{\infty}\left(0, T ; H^{k-i}(\Omega)\right)}<+\infty\right\} .
$$

We obtain the following convergence result.

Theorem 3 Let $\varphi$ satisfying (3.9). Let $\left(E_{0}, H_{0}\right)$ be the solution to (3.5)-(3.6)-(3.7) which lifespan is denoted by $T^{*}$. We assume that for all $T<T^{*}, E_{0} \in \mathcal{H}^{7}\left(\Omega_{T}\right)$. For $\varepsilon>0$, let $\left(E_{\varepsilon}, H_{\varepsilon}, v_{\varepsilon}\right)$ be the solution to (3.1)(3.2)-(3.3) which lifespan is denoted by $T_{\varepsilon}^{*}$. We fix $T<T^{*}$. There exists $\varepsilon_{0}>0$ such that

$$
\forall \varepsilon<\varepsilon_{0}, T_{\varepsilon}^{*} \geq T,
$$

and there exists a constant $C$ such that for all $\varepsilon<\varepsilon_{0}$,

$$
\left\|E_{\varepsilon}-E_{0}\right\|_{\mathcal{H}^{3}\left(\Omega_{T}\right)}+\left\|H_{\varepsilon}-H_{0}\right\|_{\mathcal{H}^{3}\left(\Omega_{T}\right)}+\left\|v_{\varepsilon}\right\|_{\mathcal{H}^{3}\left(\Omega_{T}\right)} \leq C \varepsilon .
$$

As remarked in the introduction, we don't expect boundary layer formation in the IBVP (3.1)-(3.2)-(3.3) near the profile $\left(E_{0}, H_{0}, 0\right)$. Hence we use a Hilbert expansion to describe the behavior of the solution as $\varepsilon$ tends to zero.

\section{$3.2 \quad$ Hilbert expansion.}

We denote by $\rho_{\varepsilon}={ }^{t}\left(R_{\varepsilon}, S_{\varepsilon}, s_{\varepsilon}\right)$ the remainder term in the Hilbert expansion of $W_{\varepsilon}$ :

$$
\begin{aligned}
& E_{\varepsilon}=E_{0}+\varepsilon R_{\varepsilon}, \\
& H_{\varepsilon}=H_{0}+\varepsilon S_{\varepsilon}, \\
& v_{\varepsilon}=\varepsilon s_{1}+\varepsilon s_{\varepsilon},
\end{aligned}
$$

with $s_{1}=-E_{0} \partial_{t} E_{0}$. Using (3.5)-(3.6)-(3.7) and (3.1)-(3.2)-(3.3), the rest term $\rho_{\varepsilon}$ satisfies the following system:

$$
A_{0}(t, x) \partial_{t} \rho_{\varepsilon}+\sum_{j=1}^{3} A_{j} \partial_{j} \rho_{\varepsilon}+L(t, x) \rho_{\varepsilon}+B(t, x)+G\left(t, x, R_{\varepsilon}\right)+F\left(t, x, \tilde{\rho}_{\varepsilon}\right) \partial_{t} \tilde{\rho}_{\varepsilon}=-{ }^{t}\left(0,0, \frac{2}{\varepsilon} s_{\varepsilon}\right),
$$

where

$$
\begin{aligned}
& \text { - } \tilde{\rho}_{\varepsilon}=\left(\begin{array}{c}
R_{\varepsilon} \\
0 \\
s_{\varepsilon}
\end{array}\right) \\
& \text { - } A_{0}(t, x)=\left(\begin{array}{ccc}
\left(1+\left|E_{0}\right|^{2}\right) I_{3}+2 E_{0}{ }^{t} E_{0} & 0 & 2 E_{0} \\
0 & I_{3} & 0 \\
2{ }^{t} E_{0} & 0 & 2
\end{array}\right) \text {, } \\
& \text { - } L(t, x) \rho_{\varepsilon}=\left(\begin{array}{c}
2 s_{\varepsilon} \partial_{t} E_{0}+2 E_{0} \cdot R_{\varepsilon} \partial_{t} E_{0}+2\left(E_{0}{ }^{t} R_{\varepsilon}+R_{\varepsilon}{ }^{t} E_{0}\right) \partial_{t} E_{0}+2 \varepsilon \partial_{t} s_{1} R_{\varepsilon} \\
0 \\
2{ }^{t} R_{\varepsilon} \partial_{t} E_{0}
\end{array}\right) \text {, } \\
& \text { - } B(t, x)=\left(\begin{array}{c}
2 s_{1} \partial_{t} E_{0}+2 \partial_{t} s_{1} E_{0} \\
0 \\
2 \partial_{t} s_{1}
\end{array}\right)
\end{aligned}
$$


- $G\left(t, x, R_{\varepsilon}\right)=\left(\begin{array}{c}\varepsilon\left|R_{\varepsilon}\right|^{2} \partial_{t} E_{0}+\varepsilon R_{\varepsilon}{ }^{t} R_{\varepsilon} \partial_{t} E_{0} \\ 0 \\ 0\end{array}\right)$,
- $F\left(t, x, \tilde{\rho}_{\varepsilon}\right) \partial_{t} \tilde{\rho}_{\varepsilon}=\left(\begin{array}{c}\varepsilon\left(R_{\varepsilon}{ }^{t} E_{0}+E_{0}{ }^{t} R_{\varepsilon}\right) \partial_{t} R_{\varepsilon}+\varepsilon s_{\varepsilon} \partial_{t} R_{\varepsilon}+\varepsilon^{2} R_{\varepsilon}{ }^{t} R_{\varepsilon} \partial_{t} R_{\varepsilon}+2 \varepsilon R_{\varepsilon} \partial_{t} s_{\varepsilon}+\varepsilon^{2}\left|R_{\varepsilon}\right|^{2} \partial_{t} R_{\varepsilon} \\ 0 \\ 2 \varepsilon R_{\varepsilon} \cdot \partial_{t} R_{\varepsilon}\end{array}\right)$,

with the zero initial data

$$
\rho_{\varepsilon}(0, x)=0 \text { for } x \in \Omega,
$$

and the homogeneous boundary condition

$$
S_{\varepsilon} \times n+a\left(\left(R_{\varepsilon} \times n\right) \times n\right)=0 \text { for }(t, x) \in \mathbb{R}^{+} \times \Gamma .
$$

We remark that we introduced the one order term $\varepsilon s_{1}$ in the expansion of $v_{\varepsilon}$ to avoid a singular source term in the last equation of System (3.12).

For $t \leq T_{\varepsilon}^{*}$ we define $\varphi_{\varepsilon}$ and $\psi_{\varepsilon}$ by

$$
\begin{gathered}
\varphi_{\varepsilon}(t)=\left(\left\|\rho_{\varepsilon}(t)\right\|_{L^{2}(\Omega)}^{2}+\sum_{i \neq 1}\left\|\partial_{i} \rho_{\varepsilon}(t)\right\|_{L^{2}(\Omega)}^{2}+\sum_{i, j \neq 1}\left\|\partial_{i j} \rho_{\varepsilon}(t)\right\|_{L^{2}(\Omega)}^{2}+\sum_{i, j, k \neq 1}\left\|\partial_{i j k} \rho_{\varepsilon}(t)\right\|_{L^{2}(\Omega)}^{2}\right)^{\frac{1}{2}}, \\
\psi_{\varepsilon}(t)=\left(\left\|\partial_{1} \rho_{\varepsilon}(t)\right\|_{L^{2}(\Omega)}^{2}+\sum_{i}\left\|\partial_{1 i} \rho_{\varepsilon}(t)\right\|_{L^{2}(\Omega)}^{2}+\sum_{i, j}\left\|\partial_{1 i j} \rho_{\varepsilon}(t)\right\|_{L^{2}(\Omega)}^{2}\right)^{\frac{1}{2}} .
\end{gathered}
$$

Let us remark that $\varphi_{\varepsilon}$ measures the tangential derivatives $\partial_{0}=\partial_{t}, \partial_{2}, \partial_{3}$ and we have

$$
\left(\varphi_{\varepsilon}(t)\right)^{2}+\left(\psi_{\varepsilon}(t)\right)^{2}=\sum_{i=0}^{3}\left\|\partial_{t}^{i} \rho_{\varepsilon}(t)\right\|_{H^{3-i}(\Omega)}^{2} .
$$

We define also

$$
\begin{aligned}
& \Phi_{\varepsilon}(t)=\sup _{s \in[0, t]} \varphi_{\varepsilon}(s), \\
& \Psi_{\varepsilon}(t)=\sup _{s \in[0, t]} \psi_{\varepsilon}(s),
\end{aligned}
$$

so we have

$$
\Phi_{\varepsilon}(T)+\Psi_{\varepsilon}(T) \sim\left\|\rho_{\varepsilon}\right\|_{\mathcal{H}^{3}\left(\Omega_{T}\right)}
$$

\subsection{Proof of Theorem 3.}

We fix $T<T^{*}$ and we define $T_{\varepsilon}$ by

$$
T_{\varepsilon}=\sup \left\{t \leq T,\left\|\rho_{\varepsilon}\right\|_{\mathcal{H}^{3}\left(\Omega_{t}\right)} \leq \frac{1}{\sqrt{\varepsilon}}\right\},
$$

so by Proposition $2, T_{\varepsilon} \leq T_{\varepsilon}^{*}$.

The proof of Theorem 3 is organized as follows. In the first step, by variational methods on the system (3.12)-(3.13)-(3.14) we estimate the tangential derivatives of $\rho_{\varepsilon}$. In the second step we bound the normal derivatives of $\rho_{\varepsilon}$ by solving the last equation in (3.12) and using the divergence free conditions (3.4) and (3.8).

We will use the following classical lemma.

Lemma 2 For $k \geq 2, \mathcal{H}^{k}\left(\Omega_{T}\right)$ is an algebra. 
Since $E_{0} \in \mathcal{H}^{7}\left(\Omega_{T}\right)$, we obtain

$$
A_{0}, L, B \in \mathcal{H}^{5}\left(\Omega_{T}\right),
$$

so $A_{0}, L$ and $B$ are sufficiently regular to allow the following calculations.

For the convenience of the reader, we will omit the dependence on the index $\varepsilon$.

First step: estimates on $\varphi_{\varepsilon}$.

Let us establish the following lemma:

Lemma 3 There exists a constant $C$ such that for $t \in\left[0, T_{\varepsilon}\right]$,

$$
\begin{array}{r}
\frac{d}{d t}\left[\int_{\Omega}\left(A_{0} \rho \cdot \rho+\sum_{i \neq 1} A_{0} \partial_{i} \rho \cdot \partial_{i} \rho+\sum_{i, j \neq 1} A_{0} \partial_{i j} \rho \cdot \partial_{i j} \rho+\sum_{i, j, k \neq 1} A_{0} \partial_{i j k} \rho \cdot \partial_{i j k} \rho\right) d x\right. \\
\left.+\int_{\Omega}\left(\sum_{i, j, k \neq 1} F(t, x, \tilde{\rho}) \partial_{i j k} \tilde{\rho} \cdot \partial_{i j k} \tilde{\rho}\right) d x\right] \leq C\left(1+\varphi^{2}\right) .
\end{array}
$$

Proof. The nonlinear terms in (3.12) are bounded thanks to the following estimates, which are a straightforward consequence of Lemma 2 and (3.15): there exists a constant $C$ such that for all $\varepsilon>0$,

$$
\begin{aligned}
& \|G(t, x, R)\|_{\mathcal{H}^{3}\left(\Omega_{T_{\varepsilon}}\right)} \leq C, \\
& \left\|F(t, x, \tilde{\rho}) \partial_{t} \tilde{\rho}\right\|_{\mathcal{H}^{2}\left(\Omega_{T_{\varepsilon}}\right)} \leq C, \\
& \|F(t, x, \tilde{\rho})\|_{\mathcal{H}^{3}\left(\Omega_{T_{\varepsilon}}\right)} \leq C \sqrt{\varepsilon} .
\end{aligned}
$$

Taking the inner product of (3.12) with $\rho$ we obtain

$$
\begin{aligned}
& \frac{1}{2} \frac{d}{d t} \int_{\Omega} A_{0} \rho \cdot \rho d x-\int_{\Gamma} a((R \times n) \times n) \cdot R d x_{2} d x_{3}+\frac{2}{\varepsilon} \int_{\Omega}|s|^{2} d x= \\
& \frac{1}{2} \int_{\Omega} \partial_{t} A_{0} \rho \cdot \rho d x-\int_{\Omega} L \rho \cdot \rho d x-\int_{\Omega} B \cdot \rho d x-\int_{\Omega} G(t, x, R) \cdot \rho d x-\int_{\Omega} F(t, x, \tilde{\rho}) \partial_{t} \tilde{\rho} \cdot \rho d x .
\end{aligned}
$$

By assumption on the operator $a$, we have

$$
\int_{\Gamma} a((R \times n) \times n) \cdot R d x_{2} d x_{3} \leq 0
$$

Since $E_{0}$ is regular enough, by (3.16), we have immediately

$$
\left|\frac{1}{2} \int_{\Omega} \partial_{t} A_{0} \rho \cdot \rho d x-\int_{\Omega} L \rho \cdot \rho d x\right| \leq C(\varphi(t))^{2},
$$

and

$$
\left|\int_{\Omega} B \cdot \rho d x\right| \leq C \varphi(t)
$$

From (3.18),

$$
\left|\int_{\Omega} G(t, x, R) \cdot \rho d x\right| \leq\|G\|_{L^{2}(\Omega)}\|\rho\|_{L^{2}(\Omega)} \leq\|G\|_{\mathcal{H}^{3}\left(\Omega_{T_{\varepsilon}}\right)} \varphi(t) \leq C \varphi(t)
$$

and

$$
\left|\int_{\Omega} F(t, x, \tilde{\rho}) \partial_{t} \tilde{\rho} \cdot \rho d x\right| \leq\left\|F \partial_{t} \tilde{\rho}\right\|_{L^{2}(\Omega)}\|\rho\|_{L^{2}(\Omega)} \leq\left\|F \partial_{t} \tilde{\rho}\right\|_{\mathcal{H}^{2}\left(\Omega_{T_{\varepsilon}}\right)}\|\rho\|_{L^{2}(\Omega)} \leq C \varphi(t) .
$$


Thus we obtain the $L^{2}$ estimate:

$$
\frac{d}{d t} \int_{\Omega} A_{0} \rho \cdot \rho d x \leq C\left(1+(\varphi(t))^{2}\right)
$$

In the same way, differentiating (3.12) with respect to the tangential variables, we obtain

$$
\frac{d}{d t}\left[\int_{\Omega} \sum_{i \neq 1} A_{0} \partial_{i} \rho \cdot \partial_{i} \rho d x+\int_{\Omega} \sum_{i, j \neq 1} A_{0} \partial_{i j} \rho \cdot \partial_{i j} \rho d x\right] \leq C\left(1+(\varphi(t))^{2}\right) .
$$

For the third order derivatives, we use also the estimate:

for $i, j, k \neq 1$,

$$
\left|\int_{\Omega} \partial_{i j k}\left(F(t, x, \tilde{\rho}) \partial_{t} \tilde{\rho}\right) \cdot \partial_{i j k} \tilde{\rho} d x-\frac{1}{2} \frac{d}{d t} \int_{\Omega} F(t, x, \tilde{\rho}) \partial_{i j k} \tilde{\rho} \cdot \partial_{i j k} \tilde{\rho} d x\right| \leq C\left(1+(\varphi(t))^{2}\right),
$$

and we conclude the proof of Lemma 3 .

Let us recall that $A_{0}(t, x)=A_{0}\left(E_{0}\right)($ see $(3.12))$, so, since $E_{0}$ is smooth on $[0, T] \times \bar{\Omega}$, there exists a constant $\alpha>0$ such that for all $\xi \in \mathbb{R}^{3}$ and $(t, x) \in[0, T] \times \bar{\Omega}$,

$$
A_{0}(t, x) \xi \cdot \xi \geq \alpha|\xi|^{2} .
$$

In addition, from the last estimate in (3.18) we remark that, for all $t \in\left[0, T_{\varepsilon}\right]$,

$$
\left|\int_{\Omega} \sum_{i, j, k \neq 1} F(t, x, \tilde{\rho}) \partial_{i j k} \tilde{\rho} \cdot \partial_{i j k} \tilde{\rho} d x\right| \leq C_{1} \sqrt{\varepsilon}(\varphi(t))^{2} .
$$

So, integrating (3.17) and using (3.19) and (3.20) we obtain that, for $t \in\left[0, T_{\varepsilon}\right]$,

$$
\alpha \varphi^{2}(t)-C_{1} \sqrt{\varepsilon} \varphi^{2}(t) \leq C_{2} \int_{0}^{t}\left(1+\varphi^{2}(s)\right) d s
$$

so for $\varepsilon$ small enough,

$$
\varphi^{2}(t) \leq C \int_{0}^{t}\left(1+\varphi^{2}(s)\right) d s
$$

so by Gronwall lemma, we obtain that there exists $C$ such that for all $t \in\left[0, T_{\varepsilon}\right]$

$$
\varphi^{2}(t) \leq C
$$

\section{Second step: estimates on $\psi_{\varepsilon}$.}

In order to estimate the derivatives with respect to the normal variable $\partial_{1}$ we proceed as follows:

1. We rewrite (3.12) isolating $\partial_{1} R_{3}, \partial_{1} R_{2} \partial_{1} S_{3}$ and $\partial_{1} S_{2}$ in curl $R$ and curl $S$ :

$$
\left\{\begin{array}{l}
\partial_{1} R_{3}=\partial_{3} R_{1}+\partial_{t} S_{2} \\
\partial_{1} R_{2}=\partial_{2} R_{1}-\partial_{t} S_{3}
\end{array}\right.
$$

so from (3.21),

$$
\left\|\partial_{1} R_{3}\right\|_{L^{\infty}\left(0, T_{\varepsilon} ; L^{2}(\Omega)\right)}+\left\|\partial_{1} R_{2}\right\|_{L^{\infty}\left(0, T_{\varepsilon} ; L^{2}(\Omega)\right)} \leq C,
$$

and

$$
\left\{\begin{array}{l}
\partial_{1} S_{3}=\partial_{3} S_{1}-\left(A_{0} \partial_{t} \rho\right)_{2}-(L \rho)_{2}+M_{2}\left(t, x, \varepsilon, \tilde{\rho}, \partial_{t} \tilde{\rho}\right) \\
\partial_{1} S_{2}=\partial_{2} S_{1}+\left(A_{0} \partial_{t} \rho\right)_{3}+(L \rho)_{3}+M_{3}\left(t, x, \varepsilon, \tilde{\rho}, \partial_{t} \tilde{\rho}\right)
\end{array}\right.
$$


where, by (3.18),

$$
\left\|M_{2}\right\|_{\mathcal{H}^{2}\left(\Omega_{T_{\varepsilon}}\right)}+\left\|M_{3}\right\|_{\mathcal{H}^{2}\left(\Omega_{T_{\varepsilon}}\right)} \leq C
$$

Using also (3.21), we obtain that

$$
\left\|\partial_{1} S_{3}\right\|_{L^{\infty}\left(0, T_{\varepsilon} ; L^{2}(\Omega)\right)}+\left\|\partial_{1} S_{2}\right\|_{L^{\infty}\left(0, T_{\varepsilon} ; L^{2}(\Omega)\right)} \leq C .
$$

2. In order to estimate $\partial_{1} S_{1}, \partial_{1} R_{1}$ and $\partial_{1} s$, let us rewrite the divergence free conditions. From (3.4) and (3.8), we obtain

$$
\partial_{1} S_{1}=-\partial_{2} S_{2}-\partial_{3} S_{3},
$$

so we have

$$
\left\|\partial_{1} S_{1}\right\|_{L^{\infty}\left(0, T_{\varepsilon} ; L^{2}(\Omega)\right)} \leq C .
$$

It remains to estimate $\partial_{1} R_{1}$ and $\partial_{1} s$. From (3.4) and (3.8), we have

$$
\begin{aligned}
\operatorname{div}\left(\left(1+\left|E_{0}\right|^{2} R+2\left(E_{0} \cdot R\right) E_{0}+2 s E_{0}\right)=\right. & \tilde{N}\left(t, x, \varepsilon \tilde{\rho}, \partial_{1} \tilde{\rho}, \partial_{2} \tilde{\rho}, \partial_{3} \tilde{\rho}\right) \\
& :=-\operatorname{div}\left(2 s_{1} E_{0}+\varepsilon|R|^{2} E_{0}+2 \varepsilon\left(s_{1}+s+\left(E_{0} \cdot R\right) R\right)+\varepsilon^{2}|R|^{2} R\right) .
\end{aligned}
$$

We expend the left hand side term and we obtain

$$
\begin{array}{r}
\left(1+\left|E_{0}\right|^{2}+2\left(E_{0,1}\right)^{2}\right) \partial_{1} R_{1}+2 E_{0,1} \partial_{1} s+\tilde{\Lambda}(t, x) \rho+\tilde{\Lambda}_{2}(t, x) \partial_{2} \rho+\tilde{\Lambda}_{3}(t, x) \partial_{3} \rho \\
+2 E_{0,1} E_{0,2} \partial_{1} R_{2}+2 E_{0,1} E_{0,3} \partial_{1} R_{3}=\tilde{N}
\end{array}
$$

where $\tilde{\Lambda}(t, x), \tilde{\Lambda}_{2}(t, x)$ and $\tilde{\Lambda}_{3}(t, x)$ are linear operators.

Using (3.22) we obtain

$$
\partial_{1} R_{1}+2\left(1+\left|E_{0}\right|^{2}+2\left(E_{0,1}\right)^{2}\right)^{-1} E_{0,1} \partial_{1} s=\Lambda(t, x) \rho+\sum_{i \in\{0,2,3\}} \Lambda_{i}(t, x) \partial_{i} \rho+N\left(t, x, \varepsilon, \tilde{\rho}, \partial_{1} \tilde{\rho}, \partial_{2} \tilde{\rho}, \partial_{3} \tilde{\rho}\right)
$$

where $\Lambda(t, x)$ and the $\Lambda_{i}(t, x)$ are linear operators, and $N=\left(1+\left|E_{0}\right|^{2}+2\left(E_{0,1}\right)^{2}\right)^{-1} \tilde{N}$. By (3.18) we have

$$
\|N\|_{\mathcal{H}^{2}\left(\Omega_{T_{\varepsilon}}\right)} \leq C .
$$

3. Les us consider the last equation in (3.12):

$$
\partial_{t} s+\frac{1}{\varepsilon} s=-\partial_{t}\left(E_{0} \cdot R+s_{1}+\frac{\varepsilon}{2}|R|^{2}\right) .
$$

We differentiate this equation with respect to $x_{1}$ :

$$
\partial_{t} \partial_{1} s+\frac{1}{\varepsilon} \partial_{1} s=-\partial_{t}\left(E_{0,1} \partial_{1} R_{1}\right)-\partial_{t}\left(E_{0,2} \partial_{1} R_{2}+E_{0,3} \partial_{1} R_{3}+\partial_{1} E_{0} \cdot R+\partial_{1} s_{1}+\frac{\varepsilon}{2} \partial_{1}|R|^{2}\right),
$$

and using (3.27), we obtain

$$
\partial_{t}\left(h \partial_{1} s+b\right)+\frac{1}{\varepsilon} \partial_{1} s=0
$$

where

$$
\begin{gathered}
h=\left(1+\left|E_{0}\right|^{2}+2\left(E_{0,1}\right)^{2}\right)^{-1}\left(1+\left|E_{0}\right|^{2}\right), \\
b=E_{0,1}\left(\Lambda \rho+\sum_{i \neq 1} \Lambda_{i} \partial_{i} \rho+N\right)+E_{0,2} \partial_{1} R_{2}+E_{0,3} \partial_{1} R_{3}+\partial_{1} E_{0} \cdot R+\partial_{1} s_{1}+\frac{\varepsilon}{2} \partial_{1}|R|^{2} .
\end{gathered}
$$

We have

$$
\frac{1}{3} \leq h \leq 1,
$$

and, from (3.21), (3.28), (3.23) and (3.15),

$$
\|b\|_{L^{\infty}\left(0, T_{\varepsilon} ; L^{2}(\Omega)\right)} \leq C .
$$


We remark that $h \partial_{1} s+b=0$ at $t=0$ and solving (3.29):

$$
\partial_{1} s(t)=-\frac{b(t)}{h(t)}+\frac{1}{h(t)} \int_{0}^{t} \frac{1}{\varepsilon} \exp \left(-\int_{\sigma}^{t} \frac{1}{\varepsilon h(\tau)} d \tau\right) \frac{b(\sigma)}{h(\sigma)} d \sigma
$$

so, by (3.30) and (3.31),

$$
\left\|\partial_{1} s(t)\right\|_{L^{\infty}\left(0 ; T_{\varepsilon} ; L^{2}(\Omega)\right)} \leq C
$$

and by (3.27),

$$
\left\|\partial_{1} R_{1}\right\|_{L^{\infty}\left(0, T_{\varepsilon} ; L^{2}(\Omega)\right)} \leq C .
$$

Therefore we obtain the $\mathcal{H}^{1}$ estimate:

$$
\|\rho\|_{\mathcal{H}^{1}\left(\Omega_{T_{\varepsilon}}\right)} \leq C
$$

In order to obtain the $\mathcal{H}^{2}$ estimate on the remainder term $\rho$, first we deal with the derivative $\partial_{1 i} \rho, i \in\{0,2,3\}$. We differentiate (3.22), (3.24) and (3.26) with respect to $\partial_{i}$ and using (3.21) and (3.25) we obtain

$$
\begin{gathered}
\left\|\partial_{1 i} R_{2}\right\|_{L^{\infty}\left(0, T_{\varepsilon} ; L^{2}(\Omega)\right)}+\left\|\partial_{1 i} R_{3}\right\|_{L^{\infty}\left(0, T_{\varepsilon} ; L^{2}(\Omega)\right)} \leq C, \\
\left\|\partial_{1 i} S_{1}\right\|_{L^{\infty}\left(0, T_{\varepsilon} ; L^{2}(\Omega)\right)}+\left\|\partial_{1 i} S_{2}\right\|_{L^{\infty}\left(0, T_{\varepsilon} ; L^{2}(\Omega)\right)}+\left\|\partial_{1 i} S_{3}\right\|_{L^{\infty}\left(0, T_{\varepsilon} ; L^{2}(\Omega)\right)} \leq C .
\end{gathered}
$$

We differentiate (3.29) with respect to $\partial_{i}$ :

$$
\partial_{t}\left(h \partial_{1, i} s+\left(\partial_{i} h \partial_{1} s+\partial_{i} b\right)\right)+\frac{1}{\varepsilon} \partial_{1 i} s=0,
$$

from (3.21), (3.33), (3.15) we remark that

$$
\left\|\partial_{i} h \partial_{1} s+\partial_{i} b\right\|_{L^{\infty}\left(0, T_{\varepsilon} ; L^{2}(\Omega)\right)} \leq C
$$

so, in the previous process, we can replace $b$ by $\partial_{i} h \partial_{1} s+\partial_{i} b$, and we obtain

$$
\left\|\partial_{1 i} s\right\|_{L^{\infty}\left(0, T_{\varepsilon} ; L^{2}(\Omega)\right)} \leq C .
$$

We differentiate (3.27) with respect to $\partial_{i}$ and we use (3.28), (3.21), (3.34) to obtain

$$
\left\|\partial_{1 i} R_{1}\right\|_{L^{\infty}\left(0, T_{\varepsilon} ; L^{2}(\Omega)\right)} \leq C .
$$

Therefore, by (3.33)-(3.35) we have for $i \neq 1$,

$$
\left\|\partial_{1 i} \rho\right\|_{L^{\infty}\left(0, T_{\varepsilon} ; L^{2}(\Omega)\right)} \leq C .
$$

Now we estimate $\partial_{11} \rho$. Differentiating (3.22), (3.26) and (3.24) with respect to $\partial_{1}$, using (3.21), (3.36), (3.25) and (3.32) we obtain that

$$
\begin{gathered}
\left\|\partial_{11} R_{2}\right\|_{L^{\infty}\left(0, T_{\varepsilon} ; L^{2}(\Omega)\right)}+\left\|\partial_{11} R_{3}\right\|_{L^{\infty}\left(0, T_{\varepsilon} ; L^{2}(\Omega)\right)} \leq C, \\
\left\|\partial_{11} S_{1}\right\|_{L^{\infty}\left(0, T_{\varepsilon} ; L^{2}(\Omega)\right)}+\left\|\partial_{11} S_{2}\right\|_{L^{\infty}\left(0, T_{\varepsilon} ; L^{2}(\Omega)\right)}+\left\|\partial_{11} S_{3}\right\|_{L^{\infty}\left(0, T_{\varepsilon} ; L^{2}(\Omega)\right)} \leq C .
\end{gathered}
$$

Differentiating (3.29) and (3.27) with respect to $\partial_{1}$ and using in particular (3.36) we obtain by the same method that

$$
\left\|\partial_{11} s\right\|_{L^{\infty}\left(0, T_{\varepsilon} ; L^{2}(\Omega)\right)}+\left\|\partial_{11} R_{1}\right\|_{L^{\infty}\left(0, T_{\varepsilon} ; L^{2}(\Omega)\right)} \leq C,
$$

which conclude the $\mathcal{H}^{2}$ estimate:

$$
\|\rho\|_{\mathcal{H}^{2}\left(\Omega_{T_{\varepsilon}}\right)} \leq C .
$$

For the $\mathcal{H}^{3}$ estimate, we bound successively $\left\|\partial_{1 i j} \rho\right\|_{L^{\infty}\left(0, T_{\varepsilon} ; L^{2}(\Omega)\right)}$ for $i, j \neq 1,\left\|\partial_{11 i} \rho\right\|_{L^{\infty}\left(0, T_{\varepsilon} ; L^{2}(\Omega)\right)}$ for $i \neq 1$ and $\left\|\partial_{111} \rho\right\|_{L^{\infty}\left(0, T_{\varepsilon} ; L^{2}(\Omega)\right)}$. So we obtain that there exists a constant $C$ such that

$$
\|\rho\|_{\mathcal{H}^{3}\left(\Omega_{T_{\varepsilon}}\right)} \leq C .
$$




\section{Last step of the proof of Theorem 3 .}

We recall that we fixed $T<T^{*}$, and that we defined $T_{\varepsilon}$ by (3.15). So, either $T=T_{\varepsilon}$ or $\left\|\rho_{\varepsilon}\right\|_{\mathcal{H}^{3}\left(\Omega_{T_{\varepsilon}}\right)}=\frac{1}{\sqrt{\varepsilon}}$, which is contradictory with (3.37) for $\varepsilon$ small enough. So there exists $\varepsilon_{0}>0$ such that for $\varepsilon<\varepsilon_{0}, T_{\varepsilon}^{*} \geq T_{\varepsilon}=T$ and by (3.37), $\left\|\rho_{\varepsilon}\right\|_{\mathcal{H}^{3}\left(\Omega_{T}\right)} \leq C$, so we obtain (3.10) and (3.11) in Theorem 3 .

Remark 5 The same conclusions hold in the two-dimensional and the one-dimensional cases. In the 1-d case we can replace (3.11) by:

$$
\left\|e_{\varepsilon}-e_{0}\right\|_{\mathcal{H}^{2}\left(\Omega_{T}\right)}+\left\|h_{\varepsilon}-h_{0}\right\|_{\mathcal{H}^{2}\left(\Omega_{T}\right)}+\left\|v_{\varepsilon}\right\|_{\mathcal{H}^{2}\left(\Omega_{T}\right)} \leq C \varepsilon
$$

For the 1-d and 2-dTE cases, (3.10) is irrelevant since $T_{\varepsilon}^{*}=+\infty$ by Theorems 1 and 2.

\section{Appendix: Proof of Proposition 2.}

As in Section 2.3 the IBVP (2.4)-(2.7) is equivalent to the following system in the variable $V=(U, \chi)=$ $(E, H, \chi)$ :

$$
\begin{cases}(i) \quad(1+\chi) \partial_{t} E+\left(\partial_{t} \chi\right) E-\operatorname{curl} H=G_{1}, \\ (i i) \quad \partial_{t} H+\operatorname{curl} E=G_{2}, \\ (\text { iii }) \quad \partial_{t} \chi=\frac{1}{\varepsilon}\left(|E|^{2}-\chi\right),\end{cases}
$$

with zero initial data and homogeneous boundary condition:

$$
\begin{gathered}
V(0, x)=0, x \in \Omega, \\
H \times n+a((E \times n) \times n)=0,(t, x) \in \mathbb{R}^{+} \times \Gamma .
\end{gathered}
$$

We recall the divergence free conditions

$$
\operatorname{div} H=\operatorname{div}((1+\chi) E)=0,
$$

and the positiveness property

$$
\chi \geq 0 .
$$

The proof is based on the following iteration scheme.

First, $\chi_{k} \geq 0$ being given, we define $U_{k+1}=\left(E_{k+1}, H_{k+1}\right)$ by

$$
\left\{\begin{array}{l}
\left(1+\chi_{k}\right) \partial_{t} E_{k+1}+\left(\partial_{t} \chi_{k}\right) E_{k+1}-\operatorname{curl} H_{k+1}=G_{1}, \\
\partial_{t} H_{k+1}+\operatorname{curl} E_{k+1}=G_{2},
\end{array}\right.
$$

with zero initial data and homogeneous boundary condition

$$
\begin{gathered}
U_{k+1}(0, x)=0, x \in \Omega, \\
H_{k+1} \times n+a\left(\left(E_{k+1} \times n\right) \times n\right)=0,(t, x) \in \mathbb{R}^{+} \times \Gamma,
\end{gathered}
$$

so the divergence free conditions hold

$$
\operatorname{div} H_{k+1}=\operatorname{div}\left(\left(1+\chi_{k}\right) E_{k+1}\right)=0 .
$$

Afterwards we define $\chi_{k+1}$ solving the differential equation

$$
\partial_{t} \chi_{k+1}+\frac{1}{\varepsilon} \chi_{k+1}=\frac{1}{\varepsilon}\left|E_{k+1}\right|^{2},
$$


with the initial condition

$$
\chi_{k+1}(0, x)=0, x \in \Omega \text {. }
$$

We remark that

$$
\chi_{k+1} \geq 0 .
$$

We initialize the iteration scheme by taking $V_{0}=0$. Assuming that for any $T>0, V_{k} \in \mathcal{H}^{3}\left(\Omega_{T}\right)$ with $\partial_{t} \chi_{k} \in \mathcal{H}^{3}\left(\Omega_{T}\right)$, using the existence results for linear problems in [16], the system (4.4)-(4.5)-(4.6) admits a unique solution $U_{k+1}=\left(E_{k+1}, H_{k+1}\right) \in \mathcal{H}^{3}\left(\Omega_{T}\right)$ which satisfies the divergence free condition (4.7). So (4.8)-(4.9) admits a unique solution $\chi_{k+1} \in \mathcal{H}^{3}\left(\Omega_{T}\right)$ and by these equations $\partial_{t} \chi_{k+1} \in \mathcal{H}^{3}\left(\Omega_{T}\right)$ and $\chi_{k+1} \geq 0$.

\subsection{High-norm boundedness.}

We denote

$$
\xi_{k}(t)=\sum_{i=0}^{3}\left\|\partial_{t}^{i} U_{k}(t)\right\|_{H^{3-i}(\Omega)}
$$

and

$$
\varphi_{k}(t)=\sum_{i=0}^{3}\left\|\partial_{t}^{i} V_{k}(t)\right\|_{H^{3-i}(\Omega)}+\sum_{i=0}^{3}\left\|\partial_{t}^{i+1} \chi_{k}(t)\right\|_{H^{3-i}(\Omega)} .
$$

For $\alpha=\left(\alpha_{0}, \alpha_{2}, \alpha_{3}\right) \in \mathbb{N}^{3}$ we define the operator $\partial^{\alpha}=\partial_{t}^{\alpha_{0}} \partial_{2}^{\alpha_{2}} \partial_{3}^{\alpha_{3}}$ and we denote

$$
\eta_{k}(t)=\sum_{\alpha=\left(\alpha_{0}, \alpha_{2}, \alpha_{3}\right),|\alpha| \leq 3}\left\|\partial^{\alpha} U_{k}(t)\right\|_{L^{2}(\Omega)},
$$

that is, $\eta_{k}(t)$ measures the $L^{2}$-norm of the tangential derivatives of $U_{k}$.

Finally the source terms are estimated by

$$
\Gamma(t)=\|G\|_{\mathcal{H}^{3}\left(\Omega_{t}\right)}
$$

We introduce the time $T_{k}$ defined by

$$
T_{k}=\max \left\{t \geq 0, \sup _{s \in[0, t]} \varphi_{k}(s) \leq 1, \Gamma(t) \leq 1\right\} .
$$

To obtain the boundedness, we shall prove that there exists $\tilde{T}>0$ such that

$$
\forall k \geq 0, T_{k} \geq \tilde{T} .
$$

In order to estimate the tangential derivatives of $U_{k}$ we first establish the following result.

Lemma 4 There exists a constant $K>0$ such that, for all $k$, we have

$$
\forall t \leq T_{k}, \quad \frac{d}{d t} \int_{\Omega} \sum_{\alpha=\left(\alpha_{0}, \alpha_{2}, \alpha_{3}\right),|\alpha| \leq 3}\left(\left(1+\chi_{k}\right)\left|\partial^{\alpha} E_{k+1}\right|^{2}+\left|\partial^{\alpha} H_{k+1}\right|^{2}\right) d x \leq K\left(1+\left(\xi_{k+1}\right)^{2}\right) .
$$

Proof. Taking the inner product of (4.4) with $U_{k+1}$ we obtain

$$
\begin{aligned}
\frac{1}{2} \frac{d}{d t} \int_{\Omega}\left(\left(1+\chi_{k}\right)\left|E_{k+1}\right|^{2}+\left|H_{k+1}\right|^{2}\right) & d x-\int_{\partial \Omega} a\left(\left(E_{k+1} \times n\right) \times n\right) \cdot E_{k+1} d x_{2} d x_{3} \\
= & -\frac{1}{2} \int_{\Omega} \partial_{t} \chi_{k}\left|E_{k+1}\right|^{2} d x+\int_{\Omega} G \cdot U_{k+1} d x
\end{aligned}
$$

Since $\left\|\partial_{t} \chi_{k}\right\|_{L^{\infty}(\Omega)} \leq \varphi_{k}$ we get the $L^{2}$-estimate

$$
\forall t \leq T_{k}, \quad \frac{1}{2} \frac{d}{d t} \int_{\Omega}\left(\left(1+\chi_{k}\right)\left|E_{k+1}\right|^{2}+\left|H_{k+1}\right|^{2}\right) d x \leq K\left(1+\left(\xi_{k+1}\right)^{2}\right) .
$$


By the same method we obtain similar estimates concerning the tangential derivatives. Let us describe the order 3 derivatives estimates: for $\alpha=\left(\alpha_{0}, \alpha_{2}, \alpha_{3}\right)$ with $|\alpha|=\alpha_{0}+\alpha_{2}+\alpha_{3}=3$, we take the inner product of $\partial^{\alpha}(4.4)$ with $\partial^{\alpha} U_{k+1}$. We obtain

$$
\frac{1}{2} \frac{d}{d t} \int_{\Omega}\left(\left(1+\chi_{k}\right)\left|\partial^{\alpha} E_{k+1}\right|^{2}+\left|\partial^{\alpha} H_{k+1}\right|^{2}\right) d x \leq I_{1}+\ldots+I_{5},
$$

with

$$
\begin{aligned}
I_{1}=K \sum_{\substack{|\beta|=\left|\left(\beta_{0}, \beta_{2}, \beta_{3}\right)\right|=1 \\
|\gamma|=\left|\left(\gamma_{0}, \gamma_{2}, \gamma_{3}\right)\right|=3}} \int_{\Omega}\left|\partial^{\beta} \chi_{k}\left\|\partial^{\gamma} E_{k+1}\right\| \partial^{\alpha} E_{k+1}\right| d x \\
\leq K \sum_{|\beta|=1,|\gamma|=3}\left\|\partial^{\beta} \chi_{k}\right\|_{L^{\infty}(\Omega)}\left\|\partial^{\gamma} E_{k+1}\right\|_{L^{2}(\Omega)}\left\|\partial^{\alpha} E_{k+1}\right\|_{L^{2}(\Omega)}, \\
I_{2}=K \sum_{|\beta|=2,|\gamma|=2} \int_{\Omega}\left|\partial^{\beta} \chi_{k}\left\|\partial^{\gamma} E_{k+1}\right\| \partial^{\alpha} E_{k+1}\right| d x \\
\leq K \sum_{|\beta|=2,|\gamma|=2}\left\|\partial^{\beta} \chi_{k}\right\|_{L^{4}(\Omega)}\left\|\partial^{\gamma} E_{k+1}\right\|_{L^{4}(\Omega)}\left\|\partial^{\alpha} E_{k+1}\right\|_{L^{2}(\Omega)}, \\
I_{3}=K \sum_{|\beta|=3,|\gamma|=1} \int_{\Omega}\left|\partial^{\beta} \chi_{k}\left\|\partial^{\gamma} E_{k+1}\right\| \partial^{\alpha} E_{k+1}\right| d x \\
\leq K \sum_{|\beta|=3,|\gamma|=1}\left\|\partial^{\beta} \chi_{k}\right\|_{L^{2}(\Omega)}\left\|\partial^{\gamma} E_{k+1}\right\|_{L^{\infty}(\Omega)}\left\|\partial^{\alpha} E_{k+1}\right\|_{L^{2}(\Omega)}, \\
I_{4}=\int_{\Omega} \partial_{t} \partial^{\alpha} \chi_{k}\left\|E_{k+1}\right\| \partial^{\alpha} E_{k+1} \mid d x \\
\leq K\left\|\partial_{t} \partial^{\alpha} \chi_{k}\right\|_{L^{2}(\Omega)}\left\|E_{k+1}\right\|_{L^{\infty}(\Omega)}\left\|\partial^{\alpha} E_{k+1}\right\| L_{L^{2}(\Omega)}, \\
=\int_{\Omega}\left|\partial^{\alpha} G \| \partial^{\alpha} U_{k+1}\right| d x \\
\leq\left\|\partial^{\alpha} G\right\|_{L^{2}(\Omega)}\left\|\partial^{\alpha} U_{k+1}\right\|_{L^{2}(\Omega)} .
\end{aligned}
$$

So using Sobolev inequalities we obtain that for all $t \leq T_{k}$,

$$
I_{1}+\ldots+I_{5} \leq K\left(1+\left(\xi_{k+1}\right)^{2}\right) .
$$

Now we control all the derivatives of $U_{k+1}$ by the tangential derivatives with the following estimate.

Lemma 5 There exists $K>0$ such that

$$
\forall k, \forall t \leq T_{k}, \xi_{k+1}(t) \leq K\left(\eta_{k+1}(t)+\Gamma(t)\right) .
$$


Proof. In order to estimate $\partial_{1} U_{k+1}$ we rewrite (4.4) and (4.7) to obtain

$$
\begin{aligned}
& \partial_{1} H_{k+1,3}=-\partial_{t} \chi_{k} E_{k+1,2}-\left(1+\chi_{k}\right) \partial_{t} E_{k+1,2}+\partial_{3} H_{k+1,1}+G_{1,2}, \\
& \partial_{1} H_{k+1,2}=+\partial_{t} \chi_{k} E_{k+1,3}+\left(1+\chi_{k}\right) \partial_{t} E_{k+1,3}+\partial_{2} H_{k+1,1}-G_{1,3}, \\
& \partial_{1} E_{k+1,3}=\partial_{3} E_{k+1,1}+\partial_{t} H_{k+1,2}-G_{2,2}, \\
& \partial_{1} E_{k+1,2}=\partial_{2} E_{k+1,1}-\partial_{t} H_{k+1,3}+G_{2,3}, \\
& \partial_{1} H_{k+1,1}=-\partial_{2} H_{k+1,2}-\partial_{3} H_{k+1,3}, \\
& \partial_{1} E_{k+1,1}=-\partial_{2} E_{k+1,2}-\partial_{3} E_{k+1,3}-\left(1+\chi_{k}\right)^{-1}\left(\nabla \chi_{k} \cdot E_{k+1}\right) .
\end{aligned}
$$

Using (4.10), (4.11), (4.12), (4.13) and (4.14), we obtain the estimate

$$
\forall t \leq T_{k},\left\|\partial_{1} U_{k+1}\right\|_{L^{2}(\Omega)} \leq K\left(\eta_{k+1}(t)+\Gamma(t)\right) .
$$

In order to estimate $\partial_{1 i} U_{k+1}$ we differentiate (4.18) with respect to $\partial_{i}$. For $i \neq 1$, we obtain directly that

$$
\forall t \leq T_{k},\left\|\partial_{1 i} U_{k+1}\right\|_{L^{2}(\Omega)} \leq K\left(\eta_{k+1}(t)+\Gamma(t)\right),
$$

and using (4.19) we obtain

$$
\forall t \leq T_{k},\left\|\partial_{11} U_{k+1}\right\|_{L^{2}(\Omega)} \leq K\left(\eta_{k+1}(t)+\Gamma(t)\right) .
$$

With the same arguments we estimate successively $\left\|\partial_{1 i j} U_{k+1}\right\|_{L^{2}(\Omega)},\left\|\partial_{11 j} U_{k+1}\right\|_{L^{2}(\Omega)}$ and $\left\|\partial_{111} U_{k+1}\right\|_{L^{2}(\Omega)}$ where $i \neq 1$ and $j \neq 1$, so we conclude the proof of Lemma 5 .

Using (4.16) and (4.17), by Gronwall lemma, we obtain that there exists $K>0$ such that

$$
\forall k, \forall t \leq T_{k},\left(\eta_{k+1}(t)\right)^{2} \leq e^{K t}-1,
$$

so by (4.17), there exists $K>0$ such that

$$
\forall k, \forall t \leq T_{k}, \xi_{k+1}(t) \leq K\left(\left(e^{K t}-1\right)^{\frac{1}{2}}+\Gamma(t)\right) .
$$

Then, solving (4.8) we obtain that

$$
\left\|\chi_{k+1}(t)\right\|_{H^{3}(\Omega)} \leq K \sup _{s \in[0, t]}\left(\xi_{k+1}(s)\right)^{2} .
$$

Using (4.8), $\partial_{t}(4.8), \partial_{t}^{2}(4.8)$ and $\partial_{t}^{3}(4.8)$ we obtain that

$$
\sum_{i=1}^{4}\left\|\partial_{t}^{i} \chi_{k+1}\right\|_{H^{4-i}(\Omega)} \leq K \sup _{s \in[0, t]}\left(\xi_{k+1}(s)\right)^{2} .
$$

So there exists $K>0$ such that

$$
\forall k, \varphi_{k+1}(t) \leq \xi_{k+1}(t)+K \sup _{s \in[0, t]}\left(\xi_{k+1}(s)\right)^{2} .
$$

Therefore with (4.20) there exists $K>0$ such that

$$
\forall k, \forall t \leq T_{k}, \varphi_{k+1}(t) \leq g(t):=K\left(\left(e^{K t}-1\right)^{\frac{1}{2}}+\Gamma(t)+\left(\left(e^{K t}-1\right)^{\frac{1}{2}}+\Gamma(t)\right)^{2}\right) .
$$

Since $g(0)=0$ there exists $\tilde{T}>0$ such that for $t \leq \tilde{T}, g(t) \leq \frac{3}{4}$ and $\Gamma(t) \leq \frac{3}{4}$, so we conclude the proof of (4.15). In addition we have obtained the high-norm boundedness:

$$
\forall k, \sup _{[0, \tilde{T}]} \varphi_{k}(t) \leq 1 .
$$




\subsection{Low-norm contraction.}

Subtracting (4.4) for $k-1$ to the one for $k$ we have

$$
\left\{\begin{aligned}
\left(1+\chi_{k}\right) \partial_{t}\left(E_{k+1}-E_{k}\right)-\operatorname{curl}\left(H_{k+1}-H_{k}\right)= & -\partial_{t} \chi_{k}\left(E_{k+1}-E_{k}\right)+\left(\chi_{k-1}-\chi_{k}\right) \partial_{t} E_{k} \\
& +\left(\partial_{t} \chi_{k-1}-\partial_{t} \chi_{k}\right) E_{k}, \\
\partial_{t}\left(H_{k+1}-H_{k}\right)+\operatorname{curl}\left(E_{k+1}-E_{k}\right)= & 0,
\end{aligned}\right.
$$

with the zero initial data and the homogeneous boundary condition

$$
\begin{gathered}
\left(E_{k+1}-E_{k}\right)(0, x)=\left(H_{k+1}-H_{k}\right)(0, x)=0, x \in \Omega, \\
\left(H_{k+1}-H_{k}\right) \times n+a\left(\left(\left(E_{k+1}-E_{k}\right) \times n\right) \times n\right)=0,(t, x) \in \mathbb{R}^{+} \times \Gamma .
\end{gathered}
$$

Taking the inner product of (4.22) with $U_{k+1}-U_{k}$ we obtain

$$
\begin{aligned}
& \frac{1}{2} \frac{d}{d t} \int_{\Omega}\left(\left(1+\chi_{k}\right)\left|E_{k+1}-E_{k}\right|^{2}+\left|H_{k+1}-H_{k}\right|^{2}\right) d x \leq-\frac{1}{2} \int_{\Omega} \partial_{t} \chi_{k}\left|E_{k+1}-E_{k}\right|^{2} d x \\
& +\int_{\Omega}\left(\chi_{k-1}-\chi_{k}\right) \partial_{t} E_{k}\left(E_{k+1}-E_{k}\right) d x+\int_{\Omega}\left(\partial_{t} \chi_{k-1}-\partial_{t} \chi_{k}\right) E_{k} \cdot\left(E_{k+1}-E_{k}\right) d x
\end{aligned}
$$

so using (4.21)

$$
\begin{gathered}
\frac{d}{d t} \int_{\Omega}\left(\left(1+\chi_{k}\right)\left|E_{k+1}-E_{k}\right|^{2}+\left|H_{k+1}-H_{k}\right|^{2}\right) d x \\
\leq K\left(\left\|E_{k+1}-E_{k}\right\|_{L^{2}(\Omega)}^{2}+\left\|\chi_{k}-\chi_{k-1}\right\|_{L^{2}(\Omega)}^{2}+\left\|\partial_{t} \chi_{k}-\partial_{t} \chi_{k-1}\right\|_{L^{2}(\Omega)}^{2}\right) .
\end{gathered}
$$

Subtracting (4.8) for $k-2$ to the one for $k-1$ we have

$$
\partial_{t}\left(\chi_{k}-\chi_{k-1}\right)+\frac{1}{\varepsilon}\left(\chi_{k}-\chi_{k-1}\right)=\frac{1}{\varepsilon}\left(E_{k}+E_{k-1}\right) \cdot\left(E_{k}-E_{k-1}\right),
$$

so

$$
\chi_{k}-\chi_{k-1}=\int_{0}^{t} \frac{1}{\varepsilon} \exp \left(\frac{t-s}{\varepsilon}\right)\left(E_{k}+E_{k-1}\right)(s) \cdot\left(E_{k}-E_{k-1}\right)(s) d s .
$$

We introduce

$$
u_{k}(t)=\left\|U_{k+1}-U_{k}\right\|_{L^{\infty}\left(0, t ; L^{2}(\Omega)\right)},
$$

and from (4.25) and (4.21) we have

$$
\left\|\left(\chi_{k}-\chi_{k-1}\right)(t)\right\|_{L^{2}(\Omega)} \leq K u_{k-1}(t),
$$

and from (4.24)

$$
\left\|\left(\partial_{t} \chi_{k}-\partial_{t} \chi_{k-1}\right)(t)\right\|_{L^{2}(\Omega)} \leq K u_{k-1}(t) .
$$

Integrating (4.23) and using (4.26) and (4.27) we have

$$
\left(u_{k}(t)\right)^{2} \leq K t\left(u_{k}(t)\right)^{2}+K t\left(u_{k-1}(t)\right)^{2} .
$$

We fix $\bar{T} \leq \tilde{T}$ such that, in (4.28), $K \bar{T} \leq \frac{1}{4}$. So we have

$$
\forall k, \forall t \leq \bar{T}, u_{k}(t) \leq \frac{1}{\sqrt{3}} u_{k-1}(t) .
$$

Hence $U_{k}$ is a Cauchy sequence in $\mathcal{C}\left(0, \bar{T} ; L^{2}(\Omega)\right)$ and by $(4.26), \chi_{k}$ is also a Cauchy sequence in $\mathcal{C}\left(0, \bar{T} ; L^{2}(\Omega)\right)$. By standard arguments we obtain a smooth local solution to (4.1)-(4.2)-(4.3). The end of the proof of Proposition 2 is classical. 


\section{References}

[1] S. Bianchini, B. Hanouzet, R. Natalini. Asymptotic behavior of smooth solutions for partially dissipative hyperbolic systems with a convex entropy. Comm. Pure Appl. Math. 60 (2007), no. 11, 1559-1622.

[2] G. Boillat. Chocs caractéristiques. C. R. Acad. Sci. Paris Sér. A-B 274 (1972), 1018-1021.

[3] G. Carbou, B. Hanouzet. Relaxation approximation of some nonlinear Maxwell initial-boundary value problem. Commun. Math. Sci. 4 (2006), no. 2, 331-344.

[4] G. Carbou, B. Hanouzet. Relaxation approximation of some initial-boundary value problem for psystems. Commun. Math. Sci. 5 (2007), no. 1, 187-203

[5] G. Carbou, B. Hanouzet. Comportement semi-linéaire d'un système hyperbolique quasi-linéaire : le modèle de Kerr-Debye. C.R. Acad. Sci. Paris, Ser. I 343 (2006), no. 4, 243-247.

[6] G. Carbou, B. Hanouzet. Relaxation Approximation of the Kerr Model for the impedance InitialBoundary Value Problem. Discrete Contin. Dyn. Syst. 2007, Dynamical Systems and Differential Equations. Proceedings of the 6th AIMS International Conference, suppl., 212-220.

[7] G. Carbou, B. Hanouzet, R. Natalini. Semilinear Behavior for Totally Linearly Degenerate Hyperbolic Systems with Relaxation. Preprint 2008, to appear in J. Differential Equations.

[8] G.Q. Chen, C. D. Levermore, T.-P. Liu. Hyperbolic conservation laws with stiff relaxation terms and entropy. Comm. Pure Appl. Math. 47 (1994) no. 6, 787-830.

[9] R. Dautray, J.-L. Lions. Analyse mathématique et calcul numérique pour les sciences et les techniques. Collection du Commissariat à l'Energie Atomique: Série Scientifique. Masson, Paris, 1984.

[10] O. Guès. Problème mixte hyperbolique quasi-linéaire caractéristique. Comm. Partial Differential Equations 15 (1990), no. 5, 595-645.

[11] B. Hanouzet, P. Huynh. Approximation par relaxation d'un système de Maxwell non linéaire. C. $R$. Acad. Sci. Paris Sér. I Math. 330 (2000), no. 3, 193-198.

[12] B. Hanouzet, R. Natalini. Global existence of smooth solutions for partially dissipative hyperbolic systems with a convex entropy. Arch. Ration. Mech. Anal. 169 (2003), no. 2, 89-117.

[13] P. Huynh. Etudes théorique et numérique de modèles de Kerr. Thèse, Université Bordeaux 1, 1999.

[14] A. Majda. Compressible fluid flow and systems of conservation laws in several space variables. Applied Mathematical Sciences, 53. Springer-Verlag, New York, 1984.

[15] R. Natalini. Recent results on hyperbolic relaxation problems. Analysis of systems of conservation laws (Aachen, 1997). Chapman \& Hall/CRC Monogr. Surv. Pure Appl. Math.,1999.

[16] R. H. Picard, W. M. Zajaczkowski. Local existence of solutions of impedance initial-boundary value problem for non-linear Maxwell equations. Math. Methods Appl. Sci. 18 (1995), no. 3, 169-199.

[17] Y.- R. Shen. The Principles of Nonlinear Optics. Wiley Interscience, 1994.

[18] Wen-An Yong. Singular perturbations of first-order hyperbolic systems with stiff source terms. $J$. Differential Equations 155 (1999), no. 1, 89-132.

[19] R.-W. Ziolkowski, J. B. Judkins. Full wave vector Maxwell equation modeling of the self-focusing of ultrashort optical pulses in a nonlinear Kerr medium exhibiting a finite response time. J. Opt; Soc. Am. B 10 (1993), no. 2, 186-198.

[20] R.-W. Ziolkowski. The incorporation of microscopic material models into FDTD approach for ultrafast optical pulses simulations. IEEE Transactions on Antennas and Propagation 45 (1997), no. 3, 375-391. 